Relative equilibria and conserved quantities in symmetric Hamiltonian systems

Montaldi, James

2000

MIMS EPrint: 2013.45

Manchester Institute for Mathematical Sciences

School of Mathematics

The University of Manchester

\footnotetext{
Reports available from: http://eprints.maths.manchester.ac.uk/

And by contacting: The MIMS Secretary

School of Mathematics

The University of Manchester

Manchester, M13 9PL, UK
} 


\title{
RELATIVE EQUILIBRIA AND CONSERVED QUANTITIES IN SYMMETRIC HAMILTONIAN SYSTEMS
}

\author{
JAMES MONTALDI \\ Institut Non Linéaire de Nice
}

\section{Introduction}

In this introduction, we first recall the basic phase space structures involved in Hamiltonian systems, the symplectic form, the Poisson brackets and the Hamiltonian function and vector fields, and the relationship between them. Afterwards we describe a few examples of Hamiltonian systems, both of the classical 'kinetic+potential' type as well as others using the symplectic/Poisson structure more explicitly.

There are many applications of the ideas in these notes that have been investigated by different people, but which I shall not cover. The major example, the one for which classical mechanics was invented, is the gravitational $N$-body problem. But there are many others too, such as rigid bodies, coupled rigid bodies, coupled rods, underwater sea vehicles, ... not to mention the infinite dimensional systems such as water waves, fluid flow, plasmas and elasticity. The interested reader should consult the books in the list of references at the end of these notes. Note that many of the items in the list of references are not in fact referred to in the text!

\subsection{Hamilton's equations}

The archetypal Hamiltonian system describes the motion of a particle in a potential well. If the particle has mass $m$, and $V(x)$ is the potential energy at the point $x$ (in whatever Euclidean space), then Newton's laws state that

$$
m \ddot{x}=-\nabla V(x) .
$$

In the 18th century, Lagrange introduced the phase space by defining $y=\dot{x}$ and passing to a first order differential equation, and Hamilton carried this further by introducing his now-famous equations

$$
\begin{aligned}
& \dot{q}=\partial H / \partial p, \\
& \dot{p}=-\partial H / \partial q,
\end{aligned}
$$

where $q$ replaces $x$, and $p=m \dot{x}$ is the momentum, and

$$
H(q, p)=\frac{1}{2 m}|p|^{2}+V(q)
$$


is the Hamiltonian, or total energy (kinetic + potential). This first order system is equivalent to Newton's law above, as is easily checked.

The principal advantage of Lagrange/Hamilton's approach is that it is more readily generalized to systems where the configuration space is not a Euclidean space, but is a manifold. Such systems usually come about because of constraints imposed (eg in the rigid body the constraints are that the distances between any two particles is fixed, and the configuration space is then the set of rotations and translations in Euclidean 3-space).

The other advantage of the Hamilton's approach is that it lends itself to generalizations to systems that are not of the kinetic + potential type, such as the model of a system of $N$ point vortices in the plane or on a sphere, which we will see below, or Euler's equations modelling the "reduced" motion of a rigid body.

These two generalizations lead to defining the dynamics in terms of a Hamiltonian on a phase space, where the phase space has the additional structure of being a Poisson or a symplectic manifold. The 'canonical' Poisson structure is given by

$$
\{f, g\}=\sum_{j=1}^{n} \frac{\partial f}{\partial p_{j}} \frac{\partial g}{\partial q_{j}}-\frac{\partial g}{\partial p_{j}} \frac{\partial f}{\partial q_{j}},
$$

where $f$ and $g$ are any two smooth functions on the phase space. The canonical symplectic structure is given by,

$$
\omega=\sum_{j=1}^{n} d p_{j} \wedge d q_{j}=d \alpha,
$$

where $\alpha=\sum_{j=1}^{n} p_{j} \wedge d q_{j}$ is the canonical Liouville 1 -form. ${ }^{a}$

We shall see other examples of Poisson and symplectic structures in the course of these lectures.

The Hamiltonian vector field $X_{H}$ is determined by the Hamiltonian $H$, a smooth function on the phase space, in either of the following ways:

$$
\begin{aligned}
\dot{F} & =\{H, F\} \\
\omega\left(v, X_{H}\right) & =d H(v),
\end{aligned}
$$

where $\dot{F}=X_{H}(F)$ is the time-rate of change of the function $F$ along the trajectories of the dynamical system. Combining these two expressions gives

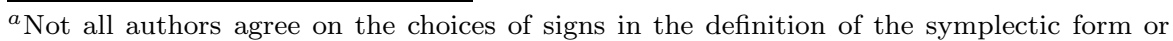
the Poisson brackets, so when using formulae involving either from a text or paper, it is necessary first to check the definitions. Our choice ensures that $X_{\{f, g\}}=\left[X_{f}, X_{g}\right]$. 
the useful formula relating the symplectic and Poisson structures,

$$
\omega\left(X_{f}, X_{g}\right)=\{f, g\}
$$

for any smooth functions $f, g$.

The first property of such Hamiltonian systems is their conservative nature: the Hamiltonian function $H$ is conserved under the dynamics and so too is the natural volume in phase space (Liouville's theorem). This has an important effect, not only on the type of dynamics encountered in such systems, but also on the types of generic bifurcations that can occur. Indeed, the first consequence of these conservation laws (energy and volume) is that one cannot have attractors in Hamiltonian systems, and in particular the notion of asymptotic stability is not available.

A further feature of Hamiltonian systems is that symmetries lead to conserved quantities. The two best-known examples of this are rotational symmetry leading to conservation of angular momentum, and translational symmetry to conservation of ordinary linear momentum. These further conserved quantities could in principle complicate the types of dynamics and bifurcations one sees. However a well defined process called reduction (or symplectic reduction) can be used to replace the symmetry and conservation laws by a family of Hamiltonian systems, parametrized by these conserved quantities, on which there are general Hamiltonian systems whose bifurcations are those expected in generic systems.

That said, there $i s$ a further complication which is that the phase space(s) for these reduced systems may be singular, or change or degenerate in a family, and we are only just beginning to understand the effects of these degenerations on the dynamics and bifurcations.

\subsection{Examples}

Spherical pendulum A spherical pendulum is a particle constrained to move on the surface of a sphere under the influence of gravity. As coordinates, one can take spherical polar coordinates $\theta, \phi(\theta$ measuring the angle with the downward vertical, and $\phi$ the angle with a fixed horizontal axis). Of course, this system has the defect of being singular at $\theta=0, \pi$. The kinetic energy is

$$
T(\mathbf{q}, \dot{\mathbf{q}})=\frac{1}{2} m \ell^{2}\left(\dot{\theta}^{2}+\sin ^{2} \theta \dot{\phi}^{2}\right),
$$

while the potential energy is $V(\mathbf{q})=m g \ell(1-\cos \theta)$. The momenta conjugate to the spherical polars are

$$
p_{\theta}=\partial \mathcal{L} / \partial \dot{\theta}=m \ell^{2} \dot{\theta}, \quad p_{\phi}=\partial \mathcal{L} / \partial \dot{\phi}=m \ell^{2} \sin ^{2} \theta \dot{\phi},
$$


where $\mathcal{L}=T-V$ is the Lagrangian, and the Hamiltonian is then

$$
H(\mathbf{q}, \mathbf{p})=\frac{1}{2 m \ell^{2}}\left(p_{\theta}^{2}+\frac{1}{\sin ^{2} \theta} p_{\phi}^{2}\right)+V(\mathbf{q}) .
$$

The equations of motion are given as usual by Hamilton's equations. In particular, one can see from these equations that the angular momentum $p_{\phi}$ about the vertical axis is conserved since $H$ is independent of $\phi$ (one has $\left.\dot{p}_{\phi}=\partial H / \partial \phi=0\right)$.

Point vortices Since the work of Helmholtz, Kirchhoff and Poincaré systems of point vortices on the plane have been widely studied as finite dimensional approximations to vorticity evolution in fluid dynamics. Small numbers of point vortices model the dynamics of concentrated regions of vorticity while large numbers can be used to approximate less concentrated regions. The equations of motion can be derived by substituting delta functions into Euler's equation for a two dimensional ideal fluid. For general surveys of planar point vortex systems see for example $[17,3,15]$.

For this system of point vortices, each vortex has a vorticity - a real nonzero number $\lambda$-and it is convenient to use complex numbers to describe the positions of the vortices. The evolution is described by the differential equation

$$
\dot{\bar{z}}_{j}=\frac{1}{2 \pi i} \sum_{k \neq j} \frac{\lambda_{k}}{z_{j}-z_{k}}
$$

where $z_{j}$ is a complex number representing the position of the $j$-th vortex. This system is Hamiltonian, with the Hamiltonian given by a pairwise interaction depending on the mutual distances:

$$
H(\mathbf{z})=-\frac{1}{4 \pi} \sum_{j<k} \lambda_{j} \lambda_{k} \log \left|z_{j}-z_{k}\right|^{2}
$$

This is clearly not of the form 'kinetic+potential'. The Poisson structure is

$$
\{f, g\}=\sum_{j} \lambda_{j}^{-1}\left(f_{z} g_{\bar{z}}-g_{z} f_{\bar{z}}\right)
$$

and the symplectic form is $\omega(\mathbf{u}, \mathbf{v})=\sum_{j} \lambda_{j}\left(u_{j} \bar{v}_{j}-\bar{u}_{j} v_{j}\right)$. Being of dimension 3 , the Euclidean symmetry has 3 conserved quantities associated to it-see equation (6.2).

A similar model can be obtained for point vortices on the sphere, providing a simple model for cyclones and hurricanes in planetary atmospheres. See Section 6. 


\subsection{Symmetry}

A transformation of the phase space $T: \mathcal{P} \rightarrow \mathcal{P}$ is a symmetry of the Hamiltonian system, if

(i) $H(T x)=H(x)$ for all $x \in \mathcal{P}$,

(ii) $T$ preserves the symplectic structure: $T^{*} \omega=\omega$, or

(ii') $T$ preserves the Poisson structure: $\{f \circ T, g \circ T\}(x)=\{f, g\}(T(x))$.

There are three basic ways that symmetries affect Hamiltonian systems:

(a) The image by $T$ of a solution is also a solution;

(b) A solution with initial point fixed by $T$ lies entirely within the set $\operatorname{Fix}(T, \mathcal{P})$, where

$$
\operatorname{Fix}(T, \mathcal{P})=\{x \in \mathcal{P} \mid T x=x\}
$$

(c) If $T$ is part of a continuous group, then the group gives rise to conserved quantities (Noether's theorem).

The first of these is clear, and in fact is also true of more generalized symmetries for which $H \circ T-H$ is constant, and $T^{*} \omega=c \omega$ ( $c$ a constant). This occurs for example for homotheties of the plane in the planar point vortex model described above. The second (b) is less obvious, but very well-known; it follows from a very simple calculation as follows. If $T x=x$ then

$$
\sigma_{t}(x)=\sigma_{t}(T x)=T \sigma_{t}(x),
$$

where $\sigma_{t}$ is the time $t$ flow associated to the Hamiltonian system, and so $\sigma_{t}(x)$ is fixed by $T$. If $T$ is part of a compact group, then not only is $\operatorname{Fix}(T, \mathcal{P})$ invariant, but it is a symplectic submanifold, and the restrictions of the Hamiltonian and the symplectic form (or Poisson structure) to $\operatorname{Fix}(T, \mathcal{P})$ is a Hamiltonian system which coincides with the restriction of the given Hamiltonian systeman exercise for the reader. This technique of restricting to fixed point spaces is sometimes called discrete reduction.

In these notes we will be concentrating on the effect of (c). The central force problem provides the basic motivating example of this.

\subsection{Central force problem}

Consider a particle of mass $m$ moving in the plane under a conservative force, whose potential depends only on the distance to the origin (a similar analysis is possible for the spherical pendulum). It is then natural 
to use polar coordinates $(r, \phi)$ which are adapted to the rotational symmetry of the problem, so that $V=V(r)$. The velocity of the particle is $\dot{\mathbf{x}}=(\dot{r} \cos \phi+(r \sin \phi) \dot{\phi}, \dot{r} \sin \phi-(r \cos \phi) \dot{\phi})$, so that the kinetic energy is

$$
T=\frac{m}{2}|\dot{\mathbf{x}}|^{2}=\frac{m}{2}\left(\dot{r}^{2}+r^{2} \dot{\phi}^{2}\right) .
$$

The Lagrangian is given by $\mathcal{L}=T-V$, and the Hamiltonian is $H=T+V$ with associated momentum variables given by $p_{r}=\partial \mathcal{L} / \partial r=m \dot{r}$ and $p_{\phi}=$ $\partial \mathcal{L} / \partial \dot{\phi}=m r^{2} \dot{\phi}$. Substituting for the velocities $\dot{r}$ and $\dot{\phi}$ in terms of the momenta determines the Hamiltonian to be:

$$
H\left(r, \phi, p_{r}, p_{\phi}\right)=\frac{1}{2 m}\left(p_{r}^{2}+\frac{1}{r^{2}} p_{\phi}^{2}\right)+V(r) .
$$

Then Hamilton's equations with respect to these variables are

$$
\begin{cases}\dot{r}=\frac{1}{m} p_{r}, & \dot{p}_{r}=-\frac{1}{m r^{3}} p_{\phi}^{2}+V^{\prime}(r) \\ \dot{\phi}=\frac{1}{m r^{2}} p_{\phi} & \dot{p}_{\phi}=0 .\end{cases}
$$

The last equation says that $p_{\phi}$ is preserved under the dynamics. In fact, $p_{\phi}$ is the angular momentum about the origin $r=0$.

Since $p_{\phi}$ is preserved, let us consider a motion with initial condition for which $p_{\phi}=\mu$. Then $\left(r, p_{r}\right)$ evolve as

$$
\left\{\begin{aligned}
\dot{r} & =\frac{1}{m} p_{r}, \\
\dot{p}_{r} & =-\frac{1}{m r^{3}} \mu^{2}+V^{\prime}(r)
\end{aligned}\right.
$$

This is in fact a Hamiltonian system, with Hamilton $H_{\mu}\left(r, p_{r}\right)$ obtained by substituting $\mu$ for $p_{\phi}$. So that

$$
H_{\mu}\left(r, p_{r}\right)=\frac{1}{2 m} p_{r}^{2}+\frac{\mu^{2}}{m r^{2}}+V(r) .
$$

This is a 1-degree of freedom problem, called the reduced system, with "effective" potential energy

$$
V_{\mu}(r)=\frac{\mu^{2}}{m r^{2}}+V(r)
$$

and for a given potential energy function $V(r)$, one can study how the behaviour of the system depends on $\mu$.

For example, with the gravitational potential $V(r)=-1 / r$, one obtains an effective potential of the form in Figure 1 below, where the fist graph shows the potential $V(r)$ as a function of $r$, while the second and third show $V_{\mu}(r)$ for increasing values of $\mu$. 
It is clear that for $\mu=0$ there is no equilibrium for the reduced system, while for $\mu>0$ there is an equilibrium, at $r_{\mu}$ satisfying $V_{\mu}^{\prime}\left(r_{\mu}\right)=0$-here $r_{\mu}=3 \mu^{2} / \mathrm{m}$. Indeed, in this example it is a stable equilibrium for the effective potential has a minimum at the $r_{\mu}$.
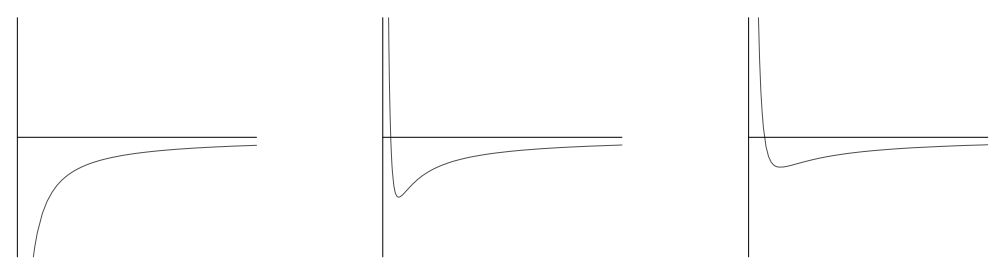

Figure 1. Effective potential for increasing values of $\mu$, for $V(r)=-1 / r$

Since $r=r_{\mu}$ and $p_{r}=0$ is an equilibrium of the reduced system, it is natural to substitute these values into the original equation (1.4). The two remaining equations are then

$$
\left\{\begin{array}{c}
\dot{\phi}=\frac{\mu}{m r_{\mu}^{2}} \\
\dot{p}_{\phi}=0 .
\end{array}\right.
$$

This describes a simple periodic orbit in the original phase space:

$$
\left(r, \phi, p_{r}, p_{\phi}\right)(t)=\left(r_{\mu}, \frac{\mu}{m r_{\mu}^{2}} t, 0, \mu\right)
$$

and there is one such periodic orbit for each value of $\mu \neq 0$ - or more in the general case if $V_{\mu}^{\prime}(r)=0$ has several solutions. In the case of a stable equilibrium in the reduced space, the nearby solutions in the phase space will be linear flows on invariant tori.

There are three things one should learn from this example:

- the reduced dynamics can vary with the conserved quantity, giving rise to bifurcation problems where the bifurcation parameter is an "internal" variable;

- the reduction is possible due to the symmetry of the original problem,

- the relationship between the equilibrium in the reduced system and the dynamic of the corresponding trajectory (relative equilibrium) in the full phase space is given by the group action. 


\subsection{Lie group actions}

These notes assume the reader has a basic knowledge of actions of Lie groups on manifolds. Here I recall a few basic formulae and properties that are used. A useful reference is the new book by Chossat and Lauterbach [5].

Let $G$ be a Lie group acting smoothly on a manifold $\mathcal{P}$, and let $\mathfrak{g}$ be its Lie algebra. We denote this action by $(g, x) \mapsto g \cdot x$. The orbit through $x$ is $G \cdot x=\{g \cdot x \mid g \in G\}$.

To each element $\xi \in \mathfrak{g}$ there is associated a vector field on $\mathcal{P}$ which we denote $\xi_{\mathcal{P}}$. It is defined as follows

$$
\xi_{\mathcal{P}}(x)=\left.\frac{d}{d t}\right|_{t=0}(\exp (t \xi) \cdot x) .
$$

The tangent space to the group orbit at $x$ is then $\mathfrak{g} \cdot x=\left\{\xi_{\mathcal{P}}(x) \mid \xi \in \mathfrak{g}\right\}$.

A simple calculation relates the vector field at $x$ with its image at $g \cdot x$ :

$$
d g_{x} \xi_{\mathcal{P}}(x)=\left(\operatorname{Ad}_{g} \xi\right)_{\mathcal{P}}(g \cdot x),
$$

where $\operatorname{Ad}_{g} \xi$ is the adjoint action of $g$ on $\xi$, which in the case of matrix groups is just

$$
\operatorname{Ad}_{g} \xi=g \xi g^{-1} .
$$

The adjoint representation of $\mathfrak{g}$ on $\mathfrak{g}$ is the infinitesimal version obtained by differentiating the adjoint action of $G$ :

$$
\operatorname{ad}_{\xi} \eta=\left.\frac{d}{d t}\right|_{t=0} \operatorname{Ad}_{\exp (t \xi)} \eta=[\xi, \eta] .
$$

Dual to the adjoint action on $\mathfrak{g}$ is the coadjoint action on $\mathfrak{g}^{*}$ :

$$
\left\langle\operatorname{Coad}_{g} \mu, \eta\right\rangle:=\left\langle\mu, \operatorname{Ad}_{g^{-1}} \eta\right\rangle,
$$

and similarly there is the infinitesimal version,

$$
\left\langle\operatorname{coad}_{\xi} \mu, \eta\right\rangle:=\left\langle\mu, \operatorname{ad}_{-\xi} \eta\right\rangle=\langle\mu,[\eta, \xi]\rangle .
$$

Examples 2.5 describe the coadjoint actions for the groups $\mathbf{S O}(3), \mathbf{S E}(2)$ and $\mathbf{S L}(2)$.

Given $x \in \mathcal{P}$, the isotropy subgroup of $x$ is

$$
G_{x}=\{g \in G \mid g \cdot x=x\} .
$$

The Lie algebra $\mathfrak{g}_{x}$ of $G_{x}$ consists of those $\xi \in \mathfrak{g}$ for which $\xi_{\mathcal{P}}(x)=0$, and the fixed point set of $K$

$$
\operatorname{Fix}(K, \mathcal{P})=\{x \in \mathcal{P} \mid K \cdot x=x\},
$$


consists of those points whose isotropy subgroup contains $K$. It is not hard to show that it's a submanifold of $\mathcal{P}$. Moreover, those points with isotropy precisely $K$ form an open (possibly empty) subset of $\operatorname{Fix}(K, \mathcal{P})$.

Stratification by orbit type If $G$ acts on a manifold $\mathcal{P}$, then the orbit space $\mathcal{P} / G$ is smooth at points where $G_{p}$ is trivial, and more generally where the orbit type in a neighbourhood of $p$ is constant.

More generally, for each subgroup $H<G$ one defines the orbit type stratum $\mathcal{P}_{(H)}$ to be the set of points $p$ for which $G_{p}$ is conjugate to $H$. This is a union of $G$-orbits, and its image in $\mathcal{P} / G$ is also called the orbit type stratum (now in the orbit space). These orbit type strata are submanifolds of $\mathcal{P}$ and $\mathcal{P} / G$, and they fit together to form a locally trivial stratification (i.e. locally it has a product structure).

For dynamical systems, the importance of this partition in to orbit type strata, is that for an equivariant vector field, the strata are preserved by the dynamics.

Slice to a group action A slice to a group action at $x \in \mathcal{P}$ is a submanifold of $\mathcal{P}$ which is transverse to the orbit through $x$ and of complementary dimension. If possible, the slice is chosen to be invariant under the isotropy subgroup $G_{x}$ (this is always possible if $G_{x}$ is compact). A basic result of the theory of Lie group actions is that under the orbit map $\mathcal{P} \rightarrow \mathcal{P} / G$ the slice projects to a neighbourhood of the image of $G \cdot x$ in the orbit space.

Principle of symmetric criticality This principle is the variational version of discrete reduction, and provides a useful method for finding critical points of invariant functions. It states that, if $G$ acts on a manifold $\mathcal{P}$, and if $f: \mathcal{P} \rightarrow \mathbf{R}$ is a smooth invariant function, then $x \in \operatorname{Fix}(G, \mathcal{P})$ is a critical point of $f$ if and only if it is a critical point of the restriction $\left.f\right|_{\operatorname{Fix}(G, \mathcal{P})}$ of $f$ to $\operatorname{Fix}(G, \mathcal{P})$. One proof is to use an invariant Riemannian metric to define an equivariant vector field $\nabla f$, which being equivariant, is tangent to $\operatorname{Fix}(G, \mathcal{P})$. For a full proof, valid also in infinite dimensions, see [58].

\section{Noether's Theorem and the Momentum Map}

The purpose of this section is to bring together facts about symmetry and conserved quantities that are useful for studying bifurcations. They are all found in various places, more or less explicitly, but not together in a single source. Furthermore, there appear to be misconceptions about whether "nonequivariant" momentum maps cause extra problems. Essentially, anything true for the equivariant ones remains true for non-equivariant ones (which are

J. Montaldi, Relative equilibria and conserved quantities .... 
in fact equivariant, but for a modified action, as we shall see below). Many of the details of this chapter can be found in the books $[16,8,10,13]$.

Examples 2.1 We will be using 3 examples of symplectic group actions in this section to illustrate various points. These arise in the models of systems of point vortices.

(A) $G=\mathbf{S O}(3)$ acts by rotations on the sphere $\mathcal{P}=S^{2}$, with symplectic form given by the usual area form with total area $4 \pi$ (for example in spherical polars, $\omega=\sin (\theta) d \theta \wedge d \phi)$. The Lie algebra $\mathfrak{s o}(3)$ can be represented by skewsymmetric matrices, and the vector field corresponding to the skew-symmetric matrix $\xi$ is simply $x \mapsto \xi x$.

(B) $G=\mathbf{S E}(2)$ acts on the plane $\mathcal{P}=\mathbf{R}^{2}$, with its usual symplectic form $\omega=$ $d x \wedge d y$. This group acts by translation and rotations; indeed, $\mathbf{S E}(2) \simeq \mathbf{R}^{2} \rtimes$ $\mathbf{S O}(2)$ (semidirect product), where $\mathbf{R}^{2}$ is the normal subgroup of translations of the plane, and $\mathbf{S O}(2)$ is the group of rotations about some point, e.g. the origin. The Lie algebra $\mathfrak{s e}(2)$ is represented by constant vector fields (corresponding to the translation subgroup) and by infinitesimal rotations.

(C) $G=\mathbf{S L}(2)=\mathbf{S L}(2, \mathbf{R})$ acts by isometries on the hyperbolic plane $\mathcal{P}=\mathcal{H}$. There are several ways to realize this action, of which perhaps the best-known is to use Möbius transformations on the upper-half plane. However, we will use one that is more in keeping with the others, which is to represent the hyperbolic plane as one sheet of a 2 -sheeted hyperboloid in $\mathbf{R}^{3}$ :

$$
\mathcal{H}=\left\{(x, y, z) \in \mathbf{R}^{3} \mid z^{2}-x^{2}-y^{2}=1, z>0\right\} .
$$

(The hyperbolic metric on $\mathcal{H}$ is induced from the Minkowski metric $\left(d x^{2}+\right.$ $\left.d y^{2}-d z^{2}\right)$ on $\mathbf{R}^{3}$.) The symplectic form on $\mathcal{H}$ is given by

$$
\omega_{\mathbf{x}}(\mathbf{u}, \mathbf{v})=-\frac{1}{2\|\mathbf{x}\|^{2}} R(\mathbf{x}) \cdot \mathbf{u} \wedge \mathbf{v}
$$

where $R(x, y, z)=(x, y,-z)$ and $\mathbf{u}, \mathbf{v} \in T_{\mathbf{x}} \mathcal{H}$. One way to realize the action of $\mathbf{S L}(2)$ on $\mathcal{H}$ is to embed $\mathcal{H}$ into the set of $2 \times 2$-trace zero matrices $\mathfrak{s l}_{2}(\mathbf{R})$ :

$$
\mathbf{x}=\left(\begin{array}{l}
x \\
y \\
z
\end{array}\right) \mapsto \hat{\mathbf{x}}=\left(\begin{array}{cc}
x & y+z \\
y-z & -x
\end{array}\right) .
$$

Then $A \cdot \hat{\mathbf{x}}=A \hat{\mathbf{x}} A^{-1}$, for $A \in \mathbf{S L}(2)$. Note that the image of the embedding consists of those matrices $X$ of trace zero, unit determinant and such that $X_{12}>X_{21}$. Under this identification, the symplectic form (2.2) becomes the Kostant-Kirillov-Souriau symplectic form on the coadjoint orbit (see Example $2.5(\mathrm{C}))$. 


\subsection{Noether's theorem}

With such a set-up, the famous theorem of Emmy Noether states that any 1-parameter group of symmetries is associated to a conserved quantity for the dynamics. In fact one needs some hypothesis such as the phase space being simply connected, or the group being semisimple (see [8] for details). For example, the circle group acting on the torus does not produce a globally well-defined conserved quantity.

How do these conserved quantities come about? We already have a procedure for passing from Hamiltonian function to Hamiltonian vector field, and here we apply the reverse procedure. For each $\xi \in \mathfrak{g}$ let $\phi_{\xi}: \mathcal{P} \rightarrow \mathbf{R}$ be a function that satisfies Hamilton's equation

$$
d \phi_{\xi}=\omega\left(\xi_{\mathcal{P}},-\right)
$$

if such a function exists. Of course each of these $\phi_{\xi}$ is only defined up to a constant, since only $d \phi_{\xi}$ is determined.

Such functions are known as momentum functions, and a symplectic action for which such momentum functions exist is said to be a Hamiltonian action. See [8] for conditions under which symplectic actions are Hamiltonian.

Theorem 2.2 (Noether) Consider a Hamiltonian action of the Lie group $G$ on the symplectic (or Poisson) manifold $\mathcal{P}$, and let $H$ be an invariant Hamiltonian. Then the flow of the Hamiltonian vector field leaves the momentum functions $\phi_{\xi}$ invariant.

Proof: A simple algebraic computation:

$$
X_{H}\left(\phi_{\xi}\right)=\left\{H, \phi_{\xi}\right\}=-\left\{\phi_{\xi}, H\right\}=-\xi_{\mathcal{P}}(H)=0 .
$$

This last equation holds because $H$ is $G$-invariant.

Momentum map We leave questions of dynamics now, and consider the structure of the set of momentum functions. The first observation is that the momentum functions $\phi_{\xi}$ can be chosen to depend linearly on $\xi$. For example, let $\left\{\xi_{1}, \ldots, \xi_{d}\right\}$ be a basis for $\mathfrak{g}$, and let $\phi_{\xi_{1}}, \ldots, \phi_{\xi_{d}}$ be Hamiltonian functions for the associated vector fields. Then for $\xi=a_{1} \xi_{1}+\cdots+a_{d} \xi_{d}$, one can put,

$$
\phi_{\xi}=a_{1} \phi_{\xi_{1}}+\cdots+a_{d} \phi_{\xi_{d}} .
$$

It is easy to check that such $\phi_{\xi}$ satisfy the necessary equation (2.4).

Thus, for each point $p \in \mathcal{P}$ we have a linear functional $\xi \mapsto \phi_{\xi}(p)$, which we call $\Phi(p)$, so that

$$
\Phi(p)=\left(\phi_{\xi_{1}}(p), \ldots, \phi_{\xi_{d}}(p)\right)
$$


is a map $\Phi: \mathcal{P} \rightarrow \mathfrak{g}^{*}$, where $\mathfrak{g}^{*}$ is the vector space dual of the Lie algebra $\mathfrak{g}$. Such a map is called a momentum map. The defining equation for the momentum map is,

$$
\left\langle d \Phi_{p}(\mathbf{v}), \xi\right\rangle=\omega_{p}\left(\xi_{\mathcal{P}}(p), \mathbf{v}\right) .
$$

for all $p \in \mathcal{P}$ all $\mathbf{v} \in T_{p} \mathcal{P}$ and all $\xi \in \mathfrak{g}$. An immediate and important consequence of $(2.5)$ is:

$$
\begin{aligned}
\operatorname{ker}\left(d \Phi_{p}\right) & =(\mathfrak{g} \cdot p)^{\omega} \\
\operatorname{im}\left(d \Phi_{p}\right) & =\mathfrak{g}_{p}^{\perp} \subset \mathfrak{g}^{*}
\end{aligned}
$$

Here, $U^{\omega}$ is the linear space that is orthogonal to $U$ with respect to the symplectic form. In particular, the momentum map is a submersion in a neighbourhood of any point where the action is free (or locally free: $\mathfrak{g}_{p}=0$ ).

The fact that $\Phi$ is only determined by the differential condition (2.5) means that it is only defined up to a constant. It follows that if $\Phi$ is a momentum map, then $\Phi_{1}$ is a momentum map if and only if there is some $C \in \mathfrak{g}^{*}$ for which, for all $p \in \mathcal{P}$,

$$
\Phi_{1}(p)=\Phi(p)+C .
$$

We return to the possibility of choosing a different $\Phi$ below.

Examples 2.3 Here we see momentum maps for three symplectic actions that arise for the point-vortex models, firstly for point vortices on the sphere, secondly for those in the plane and thirdly on the hyperbolic plane. We also give the general formula for momentum maps for coadjoint actions.

(A) Let $G=\mathbf{S O}(3)$ act diagonally on the product $\mathcal{P}=S^{2} \times \ldots \times S^{2}(N$ copies). On the $i^{\text {th }}$ factor we put the symplectic form $\omega_{i}=\lambda_{i} \omega_{0}$, where $\omega_{0}$ is the canonical area form on the unit sphere $\left(\int_{S^{2}} \omega_{0}=4 \pi\right)$. Then a momentum map is given by

$$
\Phi\left(x_{1}, \ldots, x_{N}\right)=\sum_{j} \lambda_{j} x_{j},
$$

where the Lie algebra $\mathfrak{s o}(3)$ (consisting of skew symmetric $3 \times 3$ matrices) is identified with $\mathbf{R}^{3}$ in the "usual way": $B \in \mathfrak{s o}(3)$ corresponds to $b \in \mathbf{R}^{3}$ satisfying $B u=b \times u$ for all vectors $u \in \mathbf{R}^{3}$. It is clear that this momentum map is equivariant with respect to the coadjoint action, which under this identification becomes the usual action of $\mathbf{S O}(3)$ on $\mathbf{R}^{3}$.

(B) An analogous example is $G=\mathbf{S E}(2)$ acting on a product of $N$ planes, with symplectic form $\omega=\oplus_{j} \lambda_{j} \omega_{j}$, where $\omega_{j}$ is the standard symplectic form 
on the $j^{\text {th }}$ plane. If we write $\mathbf{S E}(2)$ as $\mathbf{R}^{2} \rtimes \mathbf{S O}(2)$ (semidirect product), then the natural momentum map is

$$
\Phi\left(x_{1}, \ldots, x_{N}\right)=\left(\sum_{j} \lambda_{j} \mathbf{J} x_{j}, \frac{1}{2} \sum_{j} \lambda_{j}\left|x_{j}\right|^{2}\right) .
$$

where $\mathbf{J}=\left(\begin{array}{cc}0 & -1 \\ 1 & 0\end{array}\right)$ is the matrix for rotation through $\pi / 2$.

(C) A further analogous example is $G=\mathbf{S L}(2)$ acting on a product of $N$ copies of the hyperbolic plane, $\mathcal{P}=\mathcal{H}^{N}$, with symplectic form $\omega=\oplus_{j} \lambda_{j} \omega_{j}$, where $\omega_{j}$ is the standard symplectic form on the $j^{\text {th }}$ plane, see (2.2). The natural momentum map is given by,

$$
\Phi\left(x_{1}, \ldots, x_{N}\right)=\sum_{j} \lambda_{j} x_{j} .
$$

Remark 2.4 Consider any group acting on a manifold $X$, the configuration space. Classical mechanics of the "kinetic + potential" type takes place on the cotangent bundle of a configuration space, and in this setting the given action on $X$ induces a symplectic action on the cotangent bundle, by the formula

$$
g \cdot(x, p)=\left(g \cdot x,\left(d g_{x}\right)^{-T} p\right),
$$

where $A^{-T}$ is the inverse transpose of the operator $A$. Such actions are called cotangent actions or cotangent lifts and they always preserve the canonical symplectic form $\omega$ on the cotangent bundle.

The momentum map for cotangent actions always exists, and is given by

$$
\langle\Phi(x, p), \xi\rangle=\left\langle p, \xi_{\mathcal{P}}(x)\right\rangle,
$$

where the pairing on the right is between $T_{x}^{*} X$ and $T_{x} X$. For example, if $\mathcal{P}=T^{*} \mathbf{R}^{3}$ is the phase space for a central force problem, which has $\mathbf{S O}(3)$ symmetry, then after identifying $\mathfrak{s o}(3)^{*}$ with $\mathbf{R}^{3}$ as above, the momentum $\operatorname{map} \Phi: \mathcal{P} \rightarrow \mathfrak{s o}(3)^{*}$ is just the angular momentum.

\subsection{Equivariance of the momentum map}

A natural question arises: since a momentum map $\Phi: \mathcal{P} \rightarrow \mathfrak{g}^{*}$ is defined on a space $\mathcal{P}$ with an action of the group $G$, is there an action of $G$ on $\mathfrak{g}^{*}$ for which $\Phi$ commutes with (intertwines) the two actions? The answer was given in the affirmative by Souriau [16]. Usually, but not always, this turns out to be the coadjoint action of $G$ on $\mathfrak{g}^{*}$, and before stating Souriau's result we give some examples of coadjoint action.

J. Montaldi, Relative equilibria and conserved quantities .... 
Examples 2.5 The coadjoint actions for the groups described in Examples 2.1 are as follows.

(A) For $G=\mathbf{S O}(3)$, the Lie algebra $\mathfrak{g}=\mathfrak{s o}(3)$ consists of the $3 \times 3$ skewsymmetric matrices, and via the inner product $\langle A, B\rangle=\operatorname{tr}\left(A B^{T}\right)$ we can identify the dual $\mathfrak{g}^{*}$ with $\mathfrak{g}$. An easy computation shows that the coadjoint action is given by

$$
\operatorname{Coad}_{A} \mu=A \mu A^{T} \text {. }
$$

With the usual identification of $\mathfrak{s o}(3)$ with $\mathbf{R}^{3}$ (see Example 2.3) the coadjoint action is just the usual action of $\mathbf{S O}(3)$ by rotations, so that the orbits for the coadjoint actions are spheres centered at the origin, and the origin itself. For each of the 2 types of orbit, the isotropy subgroup is either all of $\mathbf{S O}(3)$, or it is a circle subgroup $\mathbf{S O}(2)$ of $\mathbf{S O}(3)$. This variation of the symmetry type of the orbits has interesting repercussions for the dynamics, and in particular for the families of relative equilibria.

(B) Consider now the 3-dimensional non-compact group $G=\mathbf{S E}(2)$ of Euclidean motions of the plane. As a group this is a semidirect product $\mathbf{R}^{2} \rtimes \mathbf{S O}(2)$, where $\mathbf{R}^{2}$ acts by translations and $\mathbf{S O}(2)$ by rotations about some given point (the "origin"). Elements $(\mathbf{u}, R) \in \mathbf{R}^{2} \rtimes \mathbf{S O}(2)$ can be identified with elements

$$
\left[\begin{array}{c|c}
R & \mathbf{u} \\
\hline 0 & 1
\end{array}\right] \in \mathbf{G} \mathbf{L}\left(\mathbf{R}^{2} \times \mathbf{R}\right)
$$

by introducing homogeneous coordinates. A calculation shows that the adjoint action is

$$
\operatorname{Ad}_{(\mathbf{u}, R)}(\mathbf{v}, B)=(R \mathbf{v}-B \mathbf{u}, B),
$$

since $B$ and $R$ commute. The coadjoint action is then

$$
\operatorname{Coad}_{(\mathbf{u}, R)}(\nu, \psi)=\left(R \nu, \psi+R \nu \mathbf{u}^{T}\right) .
$$

Note that since elements of $\mathfrak{s o}(2)$ are skew-symmetric, it follows that only the skew-symmetric part of $\psi+R \nu \mathbf{u}^{T}$ is relevant. One can therefore replace $\psi+R \nu \mathbf{u}^{T}$ by $\psi+\frac{1}{2}\left(R \nu \mathbf{u}^{T}-\mathbf{u} \nu^{T} R^{T}\right)$. A nice representation of this using complex numbers is given in Section 6 .

The coadjoint orbits are again of two types: first the cylinders with axis along the 1-dimensional subspace of $\mathfrak{g}^{*}$ which annihilates the translation subgroup (or its subalgebra), and secondly the individual points on that axis; that is, the points of the form $(0, \psi)$. In this case the two types of isotropy subgroup for the coadjoint action are firstly the translations in a given direction (orthogonal to $\nu$ ), so isomorphic to $\mathbf{R}$, or in the case of a single point 

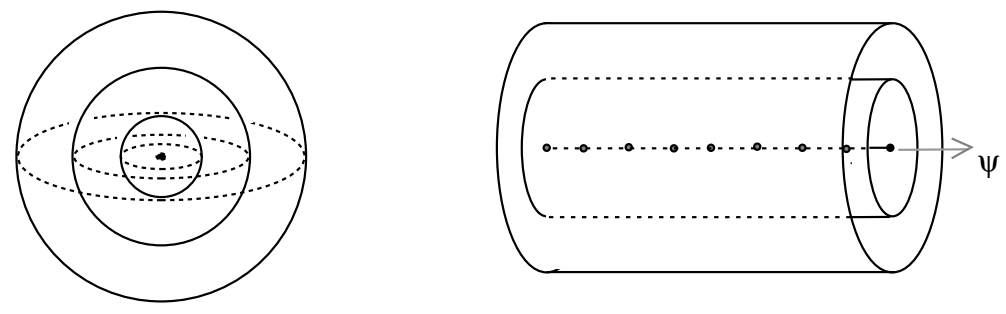

Figure 2. Coadjoint orbits for $\mathbf{S O}(3)$ and for $\mathbf{S E}(2)$

on the axis, it is the whole group. In both cases the isotropy subgroup is non-compact, a fact to be contrasted with the modified coadjoint action to be defined below.

(C) For $G=\mathbf{S L}(2)$. Let $A \in \mathbf{S L}(2)$ and $\mu \in \mathfrak{s l}(2)^{*}$, then

$$
\operatorname{Coad}_{A} \mu=A^{-T} \mu A^{T} \text {. }
$$

Notice that the determinant of the matrix in $\mathfrak{s l}(2)^{*}$ is constant on each coadjoint orbit. In fact the coadjoint orbits are of 4 types: (i) the 1-sheeted hyperboloids (where $G_{\mu} \simeq \mathbf{R}$ ), (ii) one sheet of the 2-sheeted hyperboloids (where $G_{\mu} \simeq \mathbf{S O}(2)$ ), (iii) each sheet of the cone with the origin removed (where $G_{\mu} \simeq \mathbf{R}$ ), and (iv) the origin itself (where $G_{\mu}=\mathbf{S L}(2)$ ).

It is easy to see that the momentum maps given in Examples 2.3(A,C) are equivariant with respect to the coadjoint actions described above, which is not surprising in the light of the theorem below. However this is not always true in the case of $G=\mathbf{S E}(2)$.

To describe the action that makes $\Phi$ equivariant we follow Souriau and define the cocycle

$$
\begin{aligned}
\theta: G & \rightarrow \mathfrak{g}^{*} \\
g & \mapsto \Phi(g \cdot x)-\operatorname{Coad}_{g} \Phi(x),
\end{aligned}
$$

It is of course necessary to show that this expression is independent of $x$, which it is provided $\mathcal{P}$ is connected. We leave the details to the reader: it suffices to differentiate with respect to $x$ and use the invariance of the symplectic form.

The map $\theta$ defined above allows one to define a modified coadjoint action, by

$$
\operatorname{Coad}_{g}^{\theta} \mu:=\operatorname{Coad}_{g} \mu+\theta(g) \text {. }
$$

A short calculation shows that this is indeed an action. Moreover, this action is by affine transformations whose underlying linear transformations are the coadjoint action. 
Theorem 2.6 (Souriau) Let the Lie group $G$ act on the connected symplectic manifold $\mathcal{P}$ in such a way that there is a momentum map $\Phi: \mathcal{P} \rightarrow \mathfrak{g}^{*}$. Let $\theta: G \rightarrow \mathfrak{g}^{*}$ be defined by (2.12). Then $\Phi$ is equivariant with respect to the modified action on $\mathfrak{g}^{*}$ :

$$
\Phi(g \cdot x)=\operatorname{Coad}_{g}^{\theta} \Phi(x) .
$$

Furthermore, if $G$ is either semisimple or compact then the momentum map can be chosen so that $\theta=0$.

For proofs see [16] or [8]; the first proof of equivariance in the compact case appears to be in [48].

Examples 2.7 In Examples 2.3 we gave the momentum maps for the three point-vortex models, and pointed out that for $\mathbf{S O}(3)$ and $\mathbf{S L}(2)$ the momentum map is equivariant with respect to the usual coadjoint action (not surprisingly in view of the theorem above since $\mathbf{S O}(3)$ is compact and $\mathbf{S L}(2)$ is semisimple). However, this is not always true in the planar case:

(B) Consider the momentum map given in Example 2.3(B) for the point vortex model in the plane:

$$
\Phi\left(x_{1}, \ldots, x_{N}\right)=\left(\sum_{j} \lambda_{j} \mathbf{J} x_{j}, \frac{1}{2} \sum_{j} \lambda_{j}\left|x_{j}\right|^{2}\right) .
$$

To find the action that makes this momentum map equivariant, we compute

$$
\begin{aligned}
\Phi\left(A x_{1}+\mathbf{u}, \ldots, A x_{N}+\mathbf{u}\right)= & \left(A \sum_{j} \lambda_{j} \mathbf{J} x_{j}, \frac{1}{2} \sum_{j} \lambda_{j}\left|x_{j}\right|^{2}+\sum_{j} \lambda_{j} A x_{j} . \mathbf{u}\right)+ \\
& +\Lambda\left(\mathbf{J u}, \frac{1}{2}|\mathbf{u}|^{2}\right) \\
= & \operatorname{Coad}_{(\mathbf{u}, A)} \Phi\left(x_{1}, \ldots, x_{N}\right)+\Lambda\left(\mathbf{J u}, \frac{1}{2}|\mathbf{u}|^{2}\right),
\end{aligned}
$$

where $\Lambda=\sum_{j} \lambda_{j} \in \mathbf{R}$, and Coad is given in (2.11). The cocycle associated to this momentum map is thus given by $\theta(\mathbf{u}, A)=\Lambda\left(\mathbf{J u}, \frac{1}{2}|\mathbf{u}|^{2}\right)$. If $\Lambda=0$ then $\Phi$ is equivariant with respect to the usual coadjoint action, while if $\Lambda \neq 0$ it is equivariant with respect to a modified coadjoint action. Furthermore, one can show that in this latter case there is no constant vector $C \in \mathfrak{g}^{*}$ for which $\Phi+C$ is coadjoint-equivariant. Indeed, it is enough to see that the orbits for this modified coadjoint action are in fact paraboloids, with axis the annihilator in $\mathfrak{g}^{*}$ of $\mathbf{R}^{2} \subset \mathfrak{g}$ and these are not translations of the coadjoint orbits, which are either cylinders or points. See Figure 3. Furthermore, a short calculation shows that the isotropy subgroups for this action are all compact: $G_{\mu} \simeq \mathbf{S O}(2)$, for all $\mu \in \mathfrak{s e}(2)^{*}$, which is quite different from the coadjoint action. 


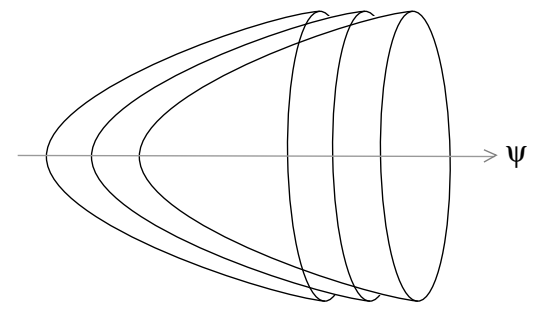

Figure 3. Modified coadjoint orbits for $\mathbf{S E}(2)$

\subsection{Reduction}

Since by Noether's theorem the dynamics preserve the level sets of the momentum map $\Phi$, it makes sense to treat the dynamical problem one level set at a time. However, these level sets are not in general symplectic submanifolds, and the system on them is therefore not a Hamiltonian system. However, it turns out that if one passes to the orbit space of one of these level sets of $\Phi$, then the resulting reduced space is symplectic, and the induced dynamics are Hamiltonian. Historically, this process of reduction was first used by Jacobi, in what is called "elimination of the nodes". However, its systematic treatment is much more recent, and is due to Meyer [47] and independently to Marsden and Weinstein [43].

As usual suppose the Lie group $G$ acts in a Hamiltonian fashion on the symplectic manifold $\mathcal{P}$, and let $\Phi: \mathcal{P} \rightarrow \mathfrak{g}^{*}$ be a momentum map which is equivariant with respect to a (possibly modified) coadjoint action as discussed above. Consider a value $\mu \in \mathfrak{g}^{*}$ of the momentum map. Then since $\Phi$ is equivariant, the isotropy subgroup $G_{\mu}$ of this modified coadjoint action acts on the level set $\Phi^{-1}(\mu)$. Define the reduced space to be

$$
\mathcal{P}_{\mu}:=\Phi^{-1}(\mu) / G_{\mu} .
$$

That is, two points of $\Phi^{-1}(\mu)$ are identified if and only if they lie in the same group orbit. This defines $\mathcal{P}_{\mu}$ as a set, but to do dynamics one needs to give it more structure.

If the group $G$ acts freely near $p$ (i.e. $G_{p}$ is trivial), then $\mathcal{P}_{\mu}$ is a smooth manifold near the image of $p$ in $\mathcal{P}_{\mu}$. This is for two reasons: firstly $\Phi$ is a submersion near $p$ by (2.7), and secondly the orbit space by $G_{\mu}$ will have no singularities. This is the case of regular reduction. We discuss singular reduction briefly below.

It is important to know whether the induced dynamics on the reduced 
spaces are also Hamiltonian. The answer of course is "yes". To see this it is necessary to define the symplectic form $\omega_{\mu}$ on $\mathcal{P}_{\mu}$. Let $\bar{u}, \bar{v} \in T_{\bar{p}} \mathcal{P}_{\mu}$, be projections of $u, v \in T_{p} \mathcal{P}$, and define

$$
\omega_{\mu}(\bar{u}, \bar{v}):=\omega(u, v) .
$$

Of course, one must show this to be well defined, non-degenerate and closed: exercises left to the reader!

The Hamiltonian is an invariant function on $\mathcal{P}$ so its restriction to $\Phi^{-1}(\mu)$ is $G_{\mu}$-invariant and so induces a well-defined function on $\mathcal{P}_{\mu}$, denoted $H_{\mu}$. The dynamics induced on the reduced space is then determined by a vector field $X_{\mu}$ which satisfies Hamilton's equation: $d H_{\mu}=\omega_{\mu}\left(X_{\mu},-\right)$.

The orbit momentum map It is clear that $\mathcal{P}_{\mu}=\Phi^{-1}(\mu) / G$ can also be defined by $\mathcal{P}_{\mu}=\Phi^{-1}\left(\mathcal{O}_{\mu}\right) / G$, where $\mathcal{O}_{\mu}$ is the coadjoint orbit through $\mu$. Since $\Phi^{-1}\left(\mathcal{O}_{\mu}\right) / G \subset \mathcal{P} / G$ it is natural to use the orbit momentum map: $\varphi: \mathcal{P} / G \rightarrow \mathfrak{g}^{*} / G$ defined by

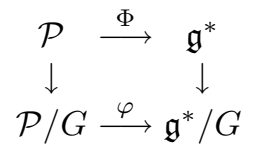

where the vertical arrows are the quotient maps. Then $\mathcal{P}_{\mu}=\varphi^{-1}\left(\mathcal{O}_{\mu}\right)$. This is very useful for studying bifurcations as the momentum value varies. However, it may not be so useful if $G_{\mu}$ is not compact, for there the orbit space $\mathfrak{g}^{*} / G$ is not in general a reasonable space (it is not even Hausdorff for example for the coadjoint action of $\mathbf{S E}(2)$ near the $\psi$-axis).

\subsection{Singular reduction}

If the action of $G$ on $\mathcal{P}$ is not free, then the reduced space is no longer a manifold. However, it was shown by Sjamaar and Lerman [67] that if $G$ is compact then the reduced space can be stratified - that is, decomposed into finitely many submanifolds which fit together in a nice way - and each stratum has a symplectic form which determines the dynamics on that stratum. In fact these strata are simply the sets of constant orbit type in $\Phi^{-1}(\mu)$, or rather their images in $\mathcal{P}_{\mu}$. For the case of a proper action of a non-compact group see [20]. The theorem follows from the local normal form of Marle and Guillemin-Sternberg $[8,42]$. 


\subsection{Symplectic slice and the reduced space}

Recall that a slice to a group action at a point $p \in \mathcal{P}$ is a submanifold $S$ through $p$ satisfying $T_{p} S \oplus \mathfrak{g} \cdot p=T_{p} \mathcal{P}$. If $G_{p}$ is compact, it can be chosen to be invariant under $G_{p}$. The slice, or more precisely $S / G_{p}$, provides a local model for the orbit space $\mathcal{P} / G$.

In the symplectic world, one needs to take into account the symplectic structure, and one wants the "symplectic slice" to provide a local model for the reduced space $\mathcal{P}_{\mu}$. In the case of free (or locally free) actions this is fairly straightforward, but in the general case, this is more delicate because the momentum map is singular. For this reason, the symplectic slice is usually taken to be a subspace of $T_{p} \mathcal{P}$ rather than a submanifold of $\mathcal{P}$.

Definition 2.8 Suppose $G_{p}$ is compact. Define $N \subset T_{p} \mathcal{P}$ to be a $G_{p}$ invariant subspace satisfying $T_{p} P=N \oplus \mathfrak{g} \cdot p$. The symplectic slice is then

$$
N_{1}:=N \cap \operatorname{ker}\left(d \Phi_{p}\right) .
$$

It follows from the implicit function theorem that local coordinates can be chosen that identify a transversal to $G_{\mu} \cdot p$ within the possibly singular set $\Phi^{-1}(\mu)$ with a subset of the symplectic slice $N_{1}$.

\section{Relative Equilibria}

An equilibrium point is a point in the phase space that is invariant under the dynamics: $p \in \mathcal{P}$ for which $X_{H}(p)=0$, or equivalently $d H_{p}=0$, and one way to define a relative equilibrium is as a group orbit that is invariant under the dynamics. Although geometrically appealing, this is not the most physically transparent definition.

Definition 3.1 A relative equilibrium is a trajectory $\gamma(t)$ in $\mathcal{P}$ such that for each $t \in \mathbf{R}$ there is a symmetry transformation $g_{t} \in G$ for which $\gamma(t)=$ $g_{t} \cdot \gamma(0)$.

In other words, the trajectory is contained in a single group orbit. It is clear that if a group orbit is invariant under the dynamics, then all the trajectories in it are relative equilibria; and conversely, if $\gamma(t)$ is the trajectory through $p$, then $g \cdot \gamma(t)$ is the trajectory through $g \cdot p$ and consequently the entire group orbit is invariant as claimed above.

For $N$-body problems in space, relative equilibria are simply motions where the shape of the body does not change, and such motions are always rigid rotations about some axis.

Proposition 3.2 Let $\Phi$ be a momentum map for the $G$-action on $\mathcal{P}$ and let $H$ be a $G$-invariant Hamiltonian on $\mathcal{P}$. Let $p \in \mathcal{P}$ and let $\mu=\Phi(p)$. Then 
the following are equivalent:

1 The trajectory $\gamma(t)$ through $p$ is a relative equilibrium,

2 The group orbit $G \cdot p$ is invariant under the dynamics,

$3 \exists \xi \in \mathfrak{g}$ such that $\gamma(t)=\exp (t \xi) \cdot p, \quad \forall t \in \mathbf{R}$,

$4 \exists \xi \in \mathfrak{g}$ such that $p$ is a critical point of $H_{\xi}=H-\phi_{\xi}$,

$5 p$ is a critical point of the restriction of $H$ to the level set $\Phi^{-1}(\mu)$.

Remarks 3.3 (i) The vector $\xi$ appearing in (3) is the angular velocity of the relative equilibrium. It is the same as the vector $\xi$ appearing in (4). The angular velocity is only unique if the action is locally free at $p$; in general it is well-defined modulo $\mathfrak{g}_{p}$.

(ii) If $\Phi^{-1}(\mu)$ is singular then it has a natural stratification (see $\S 2.4$ ), and condition (4) of the proposition should be interpreted as being a stratified critical point; that is all derivatives of $H$ along the stratum containing $p$ vanish at $p$.

(iii) Notice that (3) implies that relative equilibria cannot meandre around a group orbit, but must move in a rather rigid fashion. It follows from this equation that the trajectory is in fact a dense linear winding on a torus, at least if $G$ is compact. The dimension of the torus is at most equal to the rank of the group $G$. For $G=\mathbf{S O}(3)$, the rank is 1 , and this means that any RE that is not an equilibrium is in fact a periodic orbit.

Proof: The equivalence of (1) and (2) is outlined above.

Equivalence of (1) and (3): $(3) \Rightarrow(1)$ is clear. For the converse, since $\gamma(t)$ lies in $G \cdot p$ so its derivative $X_{H}(p)=\dot{\gamma}(0)$ lies in the tangent space $\mathfrak{g} \cdot p=T_{p}(G \cdot p)$. Let $\xi \in \mathfrak{g}$ be such that $X_{H}(p)=\xi_{\mathcal{P}}(p)$. Then by equivariance $X_{H}(g \cdot p)=\left(\operatorname{Ad}_{g} \xi\right)_{\mathcal{P}}(g \cdot p)$, see (1.6). Let $g_{t}=\exp (t \xi)$. Then since $\operatorname{Ad}_{g_{t}} \xi=\xi$,

$$
\frac{d}{d t}\left(g_{t} \cdot p\right)=\xi_{\mathcal{P}}\left(g_{t} \cdot p\right)=X_{H}\left(g_{t} \cdot p\right) .
$$

That is, $t \mapsto g_{t} \cdot p$ is the unique solution through $p$.

Equivalence of (3) and (4): (3) is equivalent to $X_{H}(p)=\xi_{\mathcal{P}}(p)$. Using the symplectic form, this is in turn equivalent to $d H(p)=d \phi_{\xi}(p)$.

Equivalence of (4) and (5): If $\Phi$ is submersive at $p$ then $\Phi^{-1}(\mu)$ is a submanifold of $\mathcal{P}$ and this is just Lagrange multipliers, since $d \phi_{\xi}=\langle d \Phi(p), \xi\rangle$. In the case that $\Phi$ is singular, the result follows from the principle of symmetric criticality (see the end of the Introduction for a statement). If $\Phi$ is singular at $p$, then by $(2.7) \mathfrak{g}_{p} \neq 0$. Consider the restriction of $H$ to $\operatorname{Fix}\left(G_{p}, \mathcal{P}\right)$. By the 
theorem of Sjamaar and Lerman, the set $\Phi^{-1}(\mu)$ is stratified by the subsets of constant orbit type - see $\S 2.4$. Consider then the set $\mathcal{P}_{G_{p}}$ of points with isotropy precisely $G_{p}$ (this an open subset of $\operatorname{Fix}\left(G_{p}, \mathcal{P}\right)$ containing $p$ ), and restrict both $\Phi$ and $H$ to this submanifold. Now $\Phi$ restricted to $\mathcal{P}_{G_{p}}$ is of constant rank, so that $\Phi^{-1}(\mu) \cap \mathcal{P}_{G_{p}}$ is a submanifold. The result then follows as before, since by the principle of symmetric criticality $H$ restricted to $\mathcal{P}_{G_{p}}$ has a critical point at $p$ if and only if $H$ has a critical point at $p$.

One of the earliest systematic investigations of relative equilibria was in a paper of Riemann where he classified all possible relative equilibria in a model of affine fluid flow that is now called the Riemann ellipsoid problem (or affine rigid body or pseudo-rigid body) - see [25] and [66] for details. The configuration space is the set of all $3 \times 3$ invertible matrices, and the symmetry group is $\mathbf{S O}(3) \times \mathbf{S O}(3)$. In that paper he identified the 6 conserved quantities and found geometric restrictions on the possible forms of relative equilibria using the fact that the momentum is conserved, and that the solutions are of the form given in (3) of the proposition above. In terms of general group actions, the geometric condition Riemann used is the following, which follows immediately from Proposition 3.2 above together with the conservation of momentum.

Corollary 3.4 Let $p \in \mathcal{P}$ be a point of a relative equilibrium, of angular velocity $\xi$, and let $\theta$ be the cocycle associated to the momentum map, then

$$
\operatorname{coad}_{\xi}^{\theta} \Phi(p)=0 .
$$

If $G$ is compact, so the adjoint and coadjoint actions can be identified and $\theta=0$ for a suitable choice of momentum map, this means that the angular velocity and momentum of a relative equilibrium commute. For example, for any system with symmetry $\mathbf{S O}(3)$ this means that at any relative equilibrium, the angular velocity and the value of the momentum are parallel.

Definition 3.5 A relative equilibrium through $p$ is said to be non-degenerate if the restriction of the Hessian $d^{2} H_{\xi}(p)$ to the symplectic slice $N_{1}$ is a nondegenerate quadratic form.

Definition 3.6 A point $\mu \in \mathfrak{g}^{*}$ is a regular point of the (modified) coadjoint action if in a neighbourhood of $\mu$ all the isotropy subgroups are conjugate.

Examples of regular points are: all points except the origin for the coadjoint action of $\mathbf{S O}(3)$, all points except the special axis for the coadjoint action of $\mathbf{S E}(2)$, and all points for the modified coadjoint action of $\mathbf{S E}(2)$ described in Example 2.7(B).

The following result, the first on the structure of the families of relative equilibria, was observed by V.I. Arnold in [2]. The proof is an application of 
the implicit function theorem.

Theorem 3.7 (Arnold) Suppose that $p$ lies on a non-degenerate relative equilibrium, with $G_{p}=0$ and $\mu=\Phi(p)$ a regular point of the (modified) coadjoint action. Then in a neighbourhood of $p$ there exists a smooth family of relative equilibria parametrized by $\mu \in \mathfrak{g}^{*}$.

Lyapounov stability A compact invariant subset $S$ of phase space is said to be Lyapounov stable if "any motion that starts nearby remains nearby", or more precisely, for every neighbourhood $V$ of $S$ there is another neighbourhood $V^{\prime} \subset V$ such that every trajectory intersecting $V^{\prime}$ is entirely contained in $V$.

A compact subset is said to be Lyapounov stable relative to or modulo $G$ if the $V$ and $V^{\prime}$ above are only required to be $G$-invariant subsets.

The principal tool for showing an equilibrium to be Lyapounov stable is Dirichlet's criterion, which is that if the equilibrium point is a non-degenerate local minimum of the Hamiltonian, then it is Lyapounov stable. The proof consists of noting that in this case the level sets of the Hamiltonian are topologically spheres surrounding the equilibrium, and so by conservation of energy, if a trajectory lies within one of these spheres, it remains within it.

It is reasonably clear firstly that it is sufficient if any conserved quantity has a local minimum at the equilibrium point, not necessarily the Hamiltonian itself, and secondly that the local minimum may in fact be degenerate. These observations lead to the notion of ...

Extremal relative equilibria A special role is played by extremal relative equilibria. This is partly due to Dirichlet's criterion for Lyapounov stability, and partly because of their robustness.

A relative equilibrium is said to be extremal if the reduced Hamiltonian $H_{\mu}$ on $\mathcal{P}_{\mu}$ has a local extremum (max or min) at that point. This is usually established by showing the restriction to the symplectic slice of the Hessian of $H_{\xi}=H-\phi_{\xi}$ to be positive (or negative) definite, for some $\xi$ for which $H_{\xi}$ has a critical point at $p$, see Proposition 3.2.

It is of course conceivable that a relative equilibrium is extremal while the Hessian matrix is degenerate. The simplest case of this is for $H(x, y)=x^{2}+y^{4}$ in the plane. In fact this arises in the case of 7 identical point vortices in the plane. The configuration where they lie at the vertices of a regular heptagon is a relative equilibrium, and the Hessian of $H$ on the symplectic slice is only positive semi-definite. However, a lengthy calculation shows that the relevant fourth order terms do not vanish, and the reduced Hamiltonian does indeed have a local minimum there.

For the following statement, recall that any momentum map is equivariant with respect to an appropriate action of $G$ on $\mathfrak{g}^{*}$ (Section 2), and for $\mu \in \mathfrak{g}^{*}$ 
we write $G_{\mu}$ for the isotropy subgroup of $\mu$ for that action.

Theorem $3.8([48,34])$ Let $G$ act properly on $\mathcal{P}$ with momentum map $\Phi$, and suppose $p \in \Phi^{-1}(\mu)$ is an extremal relative equilibrium, with $G_{\mu}$ compact. Then,

(i) The relative equilibrium is Lyapounov stable, relative to $G$;

(ii) There is a G-invariant neighbourhood $U$ of $p$ such that, for all $\mu^{\prime} \in \Phi(U)$ there is a relative equilibrium in $U \cap \Phi^{-1}\left(\mu^{\prime}\right)$.

The proof is mostly point-set topology on the orbit space and using the orbit momentum map (2.16), though part (ii) uses the deeper property of (local) openness of the momentum map. In fact the proof of (i) also holds if $\mu$ is a regular point for the (modified) coadjoint action, even if $G_{\mu}$ is not compact. By Proposition 2.4 of [35] this result can be refined to conclude that the relative equilibrium is stable relative to $G_{\mu}$, as described by Patrick [59]. Note that the compactness of $G_{\mu}$ ensures that $\mathfrak{g}_{\mu}$ has a $G_{\mu}$-invariant complement in $\mathfrak{g}$, as required in [35]. A consequence of using point-set topology is that there is very little information on the structure of the family of relative equilibria; for such information see Section 5.

The crucial remaining point is how to determine whether a given relative equilibrium is extremal. In the case of free actions this was done by the so-called energy-momentum and/or energy-Casimir methods of Arnold and Marsden and others. Recently this was extended to the general case of proper actions:

Proposition 3.9 (Lerman, Singer [35]) Let $p \in \Phi^{-1}(\mu)$ be a relative equilibrium satisfying the same hypotheses as the theorem above, and let $\xi \in \mathfrak{g}$ be any angular velocity of the $\mathrm{RE}$ as in Proposition 3.2. If the restriction to the symplectic slice of the quadratic form $d^{2} H_{\xi}$ is definite, then the $\mathrm{RE}$ is extremal, and so Lyapounov stable relative to $G_{\mu}$.

\section{Bifurcations of (relative) equilibria}

In this section we take an extremely brief look at the typical bifurcations of equilibria in families of Hamiltonian systems as a single parameter is varied. These results also apply to relative equilibria, provided the reduced space is smooth in a neighbourhood of the relative equilibrium, and failing that, it applies to the stratum containing the relative equilibrium. Bifurcations of relative equilibria near singular points of the reduced space have not been investigated systematically.

J. Montaldi, Relative equilibria and conserved quantities .... 


\subsection{One degree of freedom}

Saddle-centre bifurcation

This is the generic bifurcation of equilibria in 1 d.o.f. See Fig. 4. For $\lambda<0$ there are two equilibrium points: at $(x, y)=( \pm \sqrt{-\lambda}, 0)$, one local extremum and one saddle. As $\lambda \rightarrow 0$ these coalesce and then disappear for $\lambda>0$. One notices also a homoclinic orbit for $\lambda<0$, connecting the saddle point with itself, which can also be seen as a limit of the family of periodic orbits surrounding the stable equilibrium. In terms of eigenvalues of the associated linear system, this bifurcation can be seen as a pair of simple imaginary eigenvalues (a centre) decreases along the imaginary axis, collide at 0 and "then" emerge along the real axis (a saddle). This description is slightly misleading as the centre and saddle coexist. At the point of bifurcation, the linear system is $\left(\begin{array}{ll}0 & 1 \\ 0 & 0\end{array}\right)$; that is, it has non-zero nilpotent part.

Note that this bifurcation is compatible with an antisymplectic (i.e. timereversing) symmetry $(x, y) \rightarrow(x,-y)$.

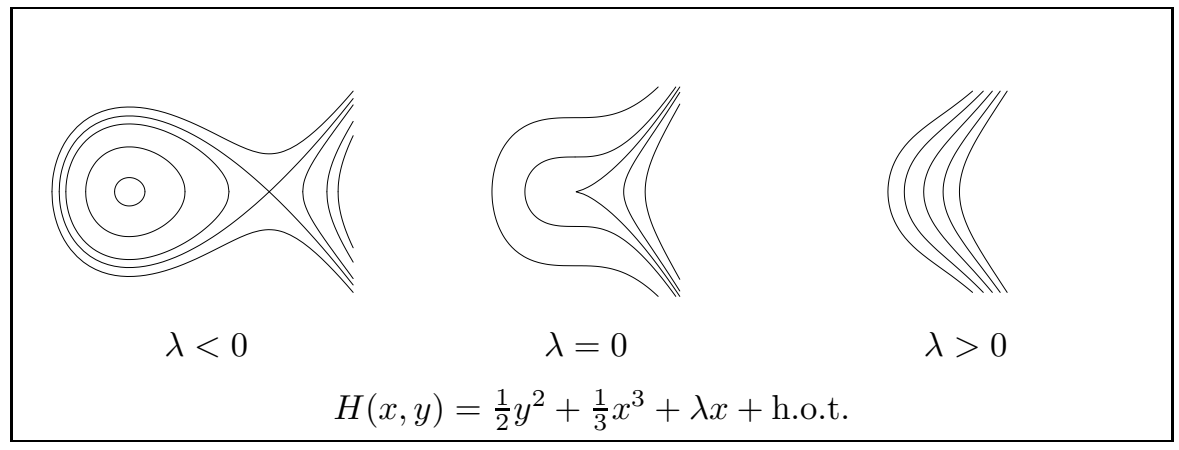

Figure 4. Saddle-centre bifurcation

Symmetric pitchfork This is usually caused by symmetry, and is 2 saddle centre bifurcations occurring simultaneously. On one side of the critical parameter value, there are 3 coexisting equilibria, while on the other side there is only one. In fact there are two types of pitchfork: a supercritical pitchfork involves two centres collapsing into a central saddle, leaving a single centre, and a subcritical pitchfork involves two saddles collapsing into a central centre, leaving a single saddle. See Figures 5 and 6 .

These results and normal forms are derived from Singularity/Catastrophe Theory. 


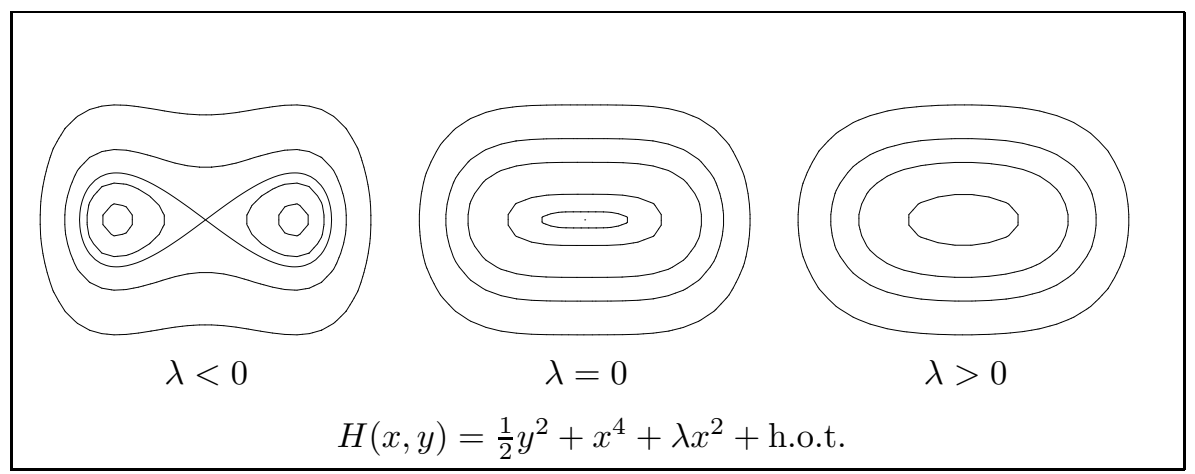

Figure 5. Supercritical Pitchfork

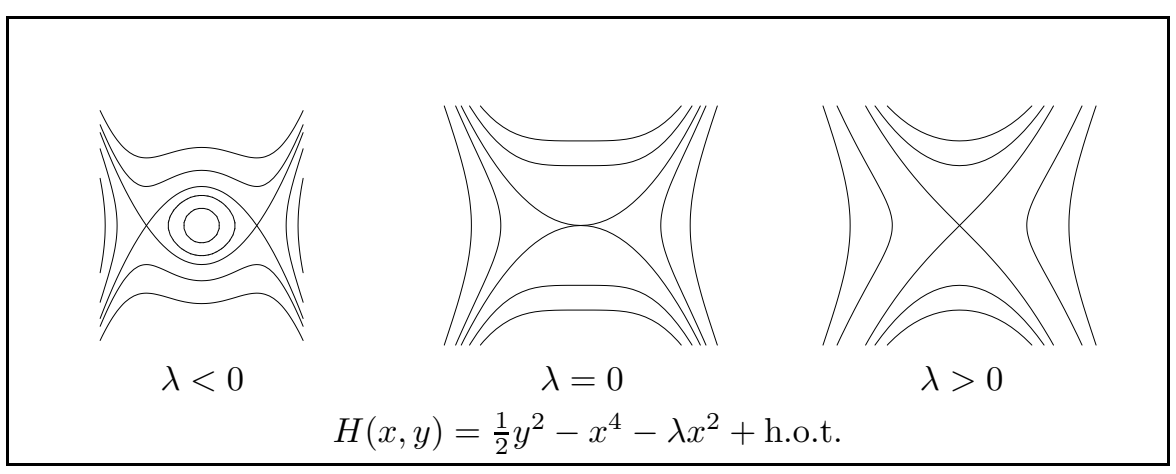

Figure 6. Subcritical Pitchfork

\subsection{Higher degrees of freedom}

From the point of view of bifurcations of equilibria, or of critical points of the Hamiltonian, the results for 1 degree of freedom carry over to higher dimensions, by adding a sum of quadratic terms in the other variables. So for example the saddle-centre bifurcations has as "normal form",

$$
H_{\lambda}(\mathbf{x}, \mathbf{y})=\frac{1}{2} y_{1}^{2}+\frac{1}{3} x_{1}^{3}+\lambda x_{1}+\frac{1}{2} \sum_{j>1}\left( \pm x_{j}^{2} \pm y_{j}^{2}\right)+\text { h.o.t. }
$$

Whether any of the equilibria are stable depends of course on the signs of the quadratic terms.

On the other hand, it is a much more subtle question as to whether any of the associated dynamics, such as the heteroclinic connections, survive this 
passage into higher dimensions. For the saddle-centre bifurcation, see [22] and for the time-reversible case, see the lectures of Eric Lombardi in this volume, and for more detail [39].

\section{Geometric Bifurcations}

In this section we discuss bifurcations of the families of relative equilibria (REs) due to degenerations in the geometry of the momentum map. One understands fairly well now the geometry of the family of relative equilibria in the neighbourhood of a point where the reduced phase spaces change in dimension, which occurs at special values of the momentum map, provided however that the group action on the phase space is (locally) free, so that the momentum map is submersive. However, the general structure of relative equilibria near points with continuous isotropy is not so well understood, although some recent progress has been made.

Notation Throughout the theorems below, we will suppose that $p \in \mathcal{P}$ is a point on a non-degenerate $\mathrm{RE}$, that the angular velocity of this $\mathrm{RE}$ is $\xi$ and the momentum value is $\mu$. Recall that an RE is said to be non-degenerate if the Hessian $d^{2} H_{\xi}$ restricted to the symplectic slice is a non-degenerate quadratic form.

In an important paper [60], George Patrick investigates the structure of the set of relative equilibria as quoted in the following theorem. He also studied the nearby dynamics and introduced the notion of drift around relative equilibria in terms of the linearized vector field there, but we will not be describing that aspect here.

Theorem 5.1 (Patrick [60]) Assume $G$ is compact, $G_{p}$ is finite and $G_{\xi} \cap$ $G_{\mu}$ is a maximal torus. Then in a neighbourhood of $p$ the set of relative equilibria forms a smooth symplectic submanifold of $\mathcal{P}$ of dimension $\operatorname{dim}(G)+$ $\operatorname{rank}(G)$.

Note that, as matrices, since $\xi$ and $\mu$ commute they are simultaneously diagonalizable. Consequently, they are both contained in a common maximal torus, so that $G_{\xi} \cap G_{\mu}$ always contains a maximal torus. The condition of the theorem is therefore a generic condition. For example, for $\mathbf{S O}(3)$ the condition is satisfied if and only if $\xi$ and $\mu$ are not both zero. This theorem has been refined by Patrick and Mark Roberts [62], where they show that assuming a generic transversality hypothesis and that as before $G_{p}$ is finite, the set of relative equilibria near $p$ is a stratified set, with the strata corresponding to the conjugacy class of the group $G_{\xi} \cap G_{\mu}$. 
The following result is more of a bifurcation theorem, as it is aimed at counting the number of relative equilibria on each reduced phase space, near a given non-degenerate RE. Recall that, if $\mu$ is a regular point for the coadjoint action and $G_{p}$ is trivial, then by Arnold's theorem (Theorem 3.7) there is a unique RE on each nearby reduced space.

Theorem 5.2 (Montaldi [48]) Assume $G_{\mu}$ is compact and $G_{p}$ trivial. Then for regular $\mu^{\prime}$ near $\mu$ there are at least $w\left(G_{\mu}\right)$ REs on the reduced space $\mathcal{P}_{\mu^{\prime}}$, where $w\left(G_{\mu}\right)$ is the order of the Weyl group of $G_{\mu}$. Furthermore, if $\xi$ is regular then there are precisely this number of relative equilibria on $\mathcal{P}_{\mu^{\prime}}$. (For non-regular $\mu^{\prime}$ see [48].)

Note that if $G_{\mu}$ is a torus, then $w\left(G_{\mu}\right)=1$, and this result reduces to Arnold's in the case that $G$ is compact. The lower bound of $w(G)$ for general $\xi$ follows from the Morse inequalities on the coadjoint orbits, and so presupposes that the REs on $\mathcal{P}_{\mu}$ are all non-degenerate. Without that assumption one can use the Lyusternik-Schnirelman category giving a lower bound of $\frac{1}{2} \operatorname{dim}\left(\mathcal{O}_{\mu}\right)+1$.

The proof of this result relies on the local normal form of Marle [42] and Guillemin-Sternberg [28]. Here I will outline the idea of the proof in the case that $\mu=0$. The idea is to use the reduced Hamiltonian rather than the augmented Hamiltonian $H_{\xi}$. So, the relative equilibria in question are critical points of the Hamiltonian restricted to $\mathcal{P}_{\mu^{\prime}}$, and near $p$ one has

$$
\mathcal{P}_{\mu^{\prime}} \simeq \mathcal{P}_{0} \times \mathcal{O}_{\mu^{\prime}} \subset \mathcal{P}_{0} \times \mathfrak{g}^{*} .
$$

The reduced space $\mathcal{P}_{0}$ can be identified with the symplectic slice $N_{1}$ (see $\S 2.5$ ), so that by hypothesis $p \in \mathcal{P}_{0}=\mathcal{P}_{0} \times\{0\}$ is a non-degenerate critical point of the restriction of $H$ to $\mathcal{P}_{0}$. Write coordinates $(y, \nu) \in \mathcal{P} \times \mathfrak{g}^{*}$. Then for each $\nu$, the function $H(\cdot, \nu)$ has an isolated non-degenerate critical point $y=y(\nu)$. Define $h: \mathfrak{g}^{*} \rightarrow \mathbf{R}$ by

$$
h(\nu)=H(y(\nu), \nu) .
$$

Then one can show that the restriction $h_{\mu^{\prime}}$ of $h$ to $\mathcal{O}_{\mu^{\prime}}$ has a critical point at $\nu$ iff $\left.H\right|_{\mathcal{P}_{\mu^{\prime}}}$ has a critical point at $(y(\nu), \nu)$. In this manner, the problem is reduced to finding critical points of the restrictions of a smooth function $h$ to coadjoint orbits $\mathcal{O}_{\mu^{\prime}}$, and then one can use Morse theory or LyusternikSchnirelman techniques.

The above proof assumes that $G_{p}$ is trivial. However, if $G_{p}$ is finite, then the proof can be modified to show that the nearby relative equilibria correspond to critical points of a smooth $G_{p^{-}}$invariant function $h$, constructed in the same manner as above, but on $\mathcal{P}_{0} \times \mathfrak{g}^{*}$ rather than on the full orbit space $\mathcal{P}_{0} \times_{G_{p}} \mathfrak{g}^{*}$. This time though, different critical points correspond to 
the same relative equilibrium if they lie in the same $G_{p}$-orbit on $\mathcal{O}_{\mu^{\prime}}$. This argument gives the following 'equivariant' version of Theorem 5.2.

Theorem 5.3 (Montaldi, Roberts [50]) If $G_{p}$ is finite, then it acts on nearby coadjoint orbits $\mathcal{O}_{\mu^{\prime}}$. If a subgroup $\Sigma$ of $G_{p}$ is such that $\operatorname{Fix}\left(\Sigma, \mathcal{O}_{\mu^{\prime}}\right)$ consists of isolated points, then they are all relative equilibria.

This result uses the fact that $H$ is $G_{p}$-invariant, and not that $G_{p}$ acts symplectically. In [50] this bifurcation result is applied to finding relative equilibria of molecules, and in [37] it is applied to finding relative equilibria of systems of point vortices on the sphere, and in both we use antisymplectic symmetries of $H$ as well as symplectic ones. An action of $G$ where the elements act either symplectically or antisymplectically, i.e. $g^{*} \omega= \pm \omega$, is said to be semisymplectic [51].

In [50], the stability of the bifurcating relative equilibria is also calculated using these methods. The reader should also see $[65,36,62,57]$ for further developments.

\section{Examples}

Let us look at three examples of symmetric Hamiltonian systems.

- Point vortices on the sphere.

- Point vortices in the plane.

- Molecules (as classical mechanical systems).

The motivation for choosing these models is that the first is relatively simple: it has no points where the action fails to be free (unless there are only 2 point vortices), and the group is compact. The bifurcations that arise are therefore of the types described in Section 5.

The second is similar, except that the group of symmetries is no longer compact.

The study of the classical mechanics of molecules is also of interest in molecular spectroscopy, where it is common practice to label particular quantum states in terms of the corresponding classical behaviour. We treat this example extremely briefly!

\subsection{Point vortices on the sphere}

The model is a finite set of point vortices on the unit sphere in 3-space. A point vortex is an infinitesimal region of vorticity in a 2-dimensional fluid flow, though we ignore the fluid, and just concentrate on the vortices. The equations of motion for this system were obtained by V.A. Bogomolov [21]. A study of the dynamics of 3 point vortices has been carried out by Kidambi

J. Montaldi, Relative equilibria and conserved quantities .... 
and Newton [30] and by Pekarsky and Marsden [63]. The case of $N$ identical point vortices has been treated in [37].

If $x_{1}, \ldots, x_{N}$ are the distinct locations of these point-vortices (unit vectors in $\mathbf{R}^{3}$ ), then the differential equation describing their motion is

$$
\dot{x}_{j}=\sum_{k \neq j} \lambda_{k} \frac{x_{k} \times x_{j}}{1-x_{k} \cdot x_{j}} .
$$

Here $\lambda_{1}, \ldots, \lambda_{N}$ are the strengths of the vortices: each $\lambda_{j}$ is a non-zero real number. It turns out that this vector field is Hamiltonian, with

$$
H(\mathbf{x})=-\frac{1}{4 \pi} \sum_{j<k} \lambda_{j} \lambda_{k} \log \left(1-x_{j} \cdot x_{k}\right),
$$

and Poisson structure

$$
\{f, g\}(\mathbf{x})=-\sum_{j} \lambda_{j}^{-1} d_{j} f \times d_{j} g \cdot x_{j},
$$

where $d_{j} f=d_{j} f(\mathbf{x}) \in \mathbf{R}^{3}$ is the differential of $f$ with respect to the point $x_{j} \in S^{2}$, and $\mathbf{x}=\left(x_{1}, \ldots, x_{N}\right)$.

The phase space is $\mathcal{P}=S^{2} \times S^{2} \times \ldots \times S^{2} \backslash \Delta$, where $\Delta$ is the 'big diagonal' where at least one pair of points coincides, which is removed to avoid collisions. The symplectic form on $\mathcal{P}$ is given by

$$
\omega=\lambda_{1} \omega_{1} \oplus \cdots \oplus \lambda_{N} \omega_{N},
$$

where $\omega_{j}$ is the standard area form on the $j^{\text {th }}$ copy of $S^{2}$.

This system has full rotational symmetry $G=\mathbf{S O}(3)$, and hence a 3component conserved quantity. After identifying $\mathfrak{s o}(3)$ with $\mathbf{R}^{3}$ as usual, this momentum map is the so-called centre of vorticity:

$$
\Phi(\mathbf{x})=\sum_{j} \lambda_{j} x_{j}
$$

There are a number of immediate general consequences for this system that can be drawn from the Hamiltonian structure. For example, if all the vorticities are of the same sign, then as $\mathbf{x} \rightarrow \Delta$ in $\mathcal{P}$, so $H(\mathbf{x}) \rightarrow+\infty$ and it follows that $H$ attains its minimum at some point; this point is necessarily an equilibrium point, and the set on which this minimum is attained is Lyapounov stable (one would expect this set to be a finite union of $\mathbf{S O}(3$ )orbits). Moreover, for any $\mu \in \mathfrak{s o}(3)^{*} \simeq \mathbf{R}^{3}$ the same argument can be applied on $\Phi^{-1}(\mu)$, and the minimum on that set is necessarily a relative equilibrium, by Proposition 3.2 . 
However, if the vorticities are of mixed signs, then there is no general statement about the existence of equilibria or relative equilibria, except for $N=3$ where all REs are known (see below).

If there are some identical vortices, then there is an extra finite symmetry group - a subgroup of the permutation group $S_{N}$. This extra finite symmetry group is used to considerable effect in [37], from which Figures 7 and 8 are taken. Moreover, the time-reversing symmetries obtained from the reflexions in $\mathbf{O}(3)$ are also used together with the principle of symmetric criticality to prove the existence of many types of relative equilibria.

For example, let $\mathbf{C}_{h}$ denote the group of order 2 generated by reflexion in the horizontal plane. Then $\mathbf{x} \in \operatorname{Fix}\left(\mathbf{C}_{h}\right)$ if all the vortices are on the equator. An application of the arguments above shows that if all the vorticities are of the same sign then there is a point on $\operatorname{Fix}\left(\mathbf{C}_{h}, \mathcal{P}\right)$ where the restriction of the Hamiltonian attains its minimum, and by the principle of symmetric criticality (see $\$ 1.5$ ) it follows that this is an equilibrium point for the full system (though no longer a local minimum in general). And the same extension to this argument as before provides relative equilibria in $\operatorname{Fix}\left(\mathbf{C}_{h}, \Phi^{-1}(\mu)\right)$. In general there will be several relative equilibria in each component of $\operatorname{Fix}\left(\mathbf{C}_{h}, \Phi^{-1}(\mu)\right)$. However, it is shown in [37] that if all the vorticities are of the same sign then in each component of $\operatorname{Fix}\left(\mathbf{C}_{h}, \mathcal{P}\right)$ there is a unique equilibrium point.

2 vortices If there are only 2 point vortices, then every solution is a relative equilibrium. Indeed, if $\Phi(\mathbf{x})=\mu \neq 0$ then the two points rotate at the same angular velocity about the axis containing $\mu$. The only possible case where $\mu=0$ is if $\lambda_{1}=\lambda_{2}$ and $x_{1}=-x_{2}$ which is an equilibrium point.

3 vortices There have been two recent studies of the system of 3 point vortices, by Kidambi and Newton [30] (who also treat the 2 vortex case in an appendix) and by Pekarsky and Marsden [63]. The former describes not only the relative equilibria, but also self-similar collapse, where triple collision occurs in finite time with the three vortices retaining their same shape up to similarity. The latter describes the REs and their stability using the techniques described in these lectures (many of which were in fact developed by Marsden and co-workers).

One of the results of Kidambi and Newton is that there are two classes of RE: those lying on a great-circle and those lying at the vertices of an equilateral triangle, and any RE belongs to one of these classes. Moreover, every equilateral triangle on the sphere is an RE, as we shall prove below.

The following results are mostly taken from [30] and [63], and some are discussed below. Denote $\lambda_{1} \lambda_{2}+\lambda_{2} \lambda_{3}+\lambda_{3} \lambda_{1}$ by $\sigma_{2}(\lambda)$.

Proposition 6.1 For the system of $N=3$ point vortices on the sphere, 

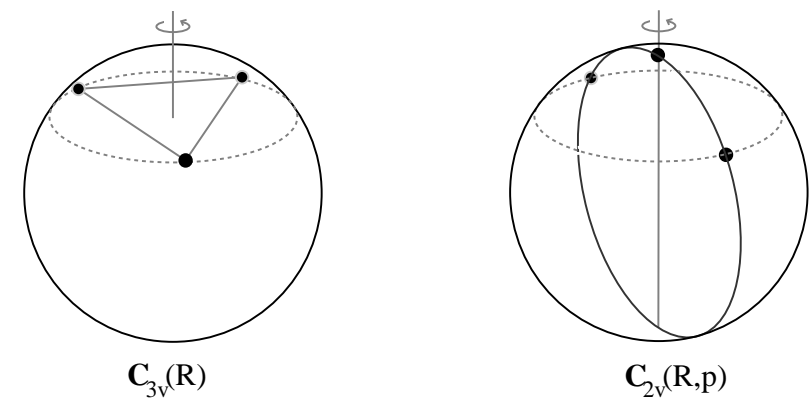

Figure 7. Relative equilibria for 3 identical vortices on the sphere

(i) There exist equilibria iff $\sigma_{2}(\lambda)>0$, and they always lie on a great circle. (ii) All equilateral configurations are $\mathrm{REs}$, and they are Lyapounov stable modulo $\mathbf{S O}(2)$ if $\sigma_{2}(\lambda)>0$, and are unstable if $\sigma_{2}(\lambda)<0$.

(iii) Self-similar collapse occurs iff $\sigma_{2}(\lambda)=0$.

(iv) The configurations where the vortices lie on a great circle, at the vertices of a right-angled isosceles triangle is always an RE. Furthermore, the triangle with $x_{1}$ at the right-angle is Lyapounov stable provided

$$
\lambda_{2}^{2}+\lambda_{3}^{2}>2 \sigma_{2}(\lambda) .
$$

The phase space is of dimension 6 in this case, and so the orbit space $\mathcal{P} / \mathbf{S O}(3)$ is of dimension 3 , and points in the orbit space correspond to the shapes of the triangle formed by the 3 vortices. The obvious set of coordinates consisting of the three pairwise distances has a problem for great circle configurations since nearby such a configuration these distances do not determine the configuration uniquely. Indeed, these three distances are $\mathbf{O}(3)$ invariants, as they do not distinguish the orientation, and configurations on a great circle have non-trivial isotropy for the $\mathbf{O}(3)$-action so it is not surprising that these coordinates have a problem there. For a good set of $\mathbf{S O}(3)$-invariants, one must use the oriented volume as well, which is what is done in the two works cited above.

However, the three distances do form a good set of coordinates away from the great circle configurations, and we will restrict our attention to those. So, let $r_{1}$ be the chord distance $\left\|x_{2}-x_{3}\right\|$ etc. Then the Hamiltonian and the orbit momentum map (2.16) are given by

$$
\begin{aligned}
H\left(r_{1}, r_{2}, r_{3}\right) & =-\frac{\lambda_{1} \lambda_{2}}{2 \pi} \log \left(r_{3}\right)-\frac{\lambda_{2} \lambda_{3}}{2 \pi} \log \left(r_{1}\right)-\frac{\lambda_{3} \lambda_{1}}{2 \pi} \log \left(r_{2}\right) \\
\varphi\left(r_{1}, r_{2}, r_{3}\right) & =|\Phi|^{2}=\left(\lambda_{1}+\lambda_{2}+\lambda_{3}\right)^{2}-\lambda_{1} \lambda_{2} r_{3}^{2}-\lambda_{2} \lambda_{3} r_{1}^{2}-\lambda_{3} \lambda_{1} r_{2}^{2}
\end{aligned}
$$


The reduced spaces are then $\mathcal{P}_{\mu}:=\varphi^{-1}\left(\mu^{2}\right)$. The relative equilibria are determined by the critical points of the restriction of $H$ to the reduced spaces, and are therefore critical points of $H-\eta \varphi$, for some $\eta$ (the Lagrange multiplier). A short calculation shows that the only solutions to this equation are

$$
r_{1}=r_{2}=r_{3}=r, \text { say, } \text { and } \eta=1 /\left(4 \pi r^{2}\right) .
$$

Thus all relative equilibria away from great circle configurations are equilateral triangles. Note that the relation between the angular velocity $\xi$ (Proposition $3.2)$ and $\eta$ is simply $\xi=2 \eta \Phi(\mathbf{x})$, since

$$
0=d(H-\eta \varphi)=d\left(H-\eta|\Phi|^{2}\right)=d H-2 \eta \Phi . d \Phi .
$$

Of course, $\Phi \neq 0$ since the vortices do not all lie on a great circle.

For the stability of these equilateral REs, we first calculate the Hessian of $H-\eta \varphi$ (this is in fact Arnold's "energy-Casimir" method):

$$
d^{2}(H-\eta \varphi)(r)=\frac{4}{r^{2}}\left(\begin{array}{ccc}
\lambda_{2} \lambda_{3} & 0 & 0 \\
0 & \lambda_{3} \lambda_{1} & 0 \\
0 & 0 & \lambda_{1} \lambda_{2}
\end{array}\right) .
$$

Now, the tangent space to $\varphi^{-1}\left(\mu^{2}\right)$ is spanned by $\left(\lambda_{1},-\lambda_{2}, 0\right),\left(\lambda_{1}, 0,-\lambda_{3}\right)$, and a computation then shows that the Hessian of the reduced Hamiltonian is definite if and only if $\sigma_{2}(\lambda)>0$.

The computations for the configurations of vortices lying on great circles are longer, and I will not go into them further here; details can be found in the original papers $[30,63]$.

Remarks 6.2 (i) The case $\sigma_{2}(\lambda)=0$ remains to be understood.

(ii) It is not known whether any other form of collapse (i.e. not self-similar) can occur.

(iii) A further bifurcation occurs at equilateral REs which lie on a great circle which to my knowledge has not been investigated.

4 vortices Much less is known in general about the case of 4 vortices. It is shown in [63] that the configuration where the four vortices lie at the vertices of a regular tetrahedron is always a relative equilibrium. It is also easy to show (from the equations of motion) that a square lying in a great circle is always an $\mathrm{RE}$, independently of the values of the vorticities. However, in contrast to the 3 -vortex case, squares not lying in great circles are not REs unless all the vortices are identical.

In the case that all the vorticities coincide, a classification of symmetric REs is given in [37], from which Figure 8 is taken. Moreover, using the implicit 


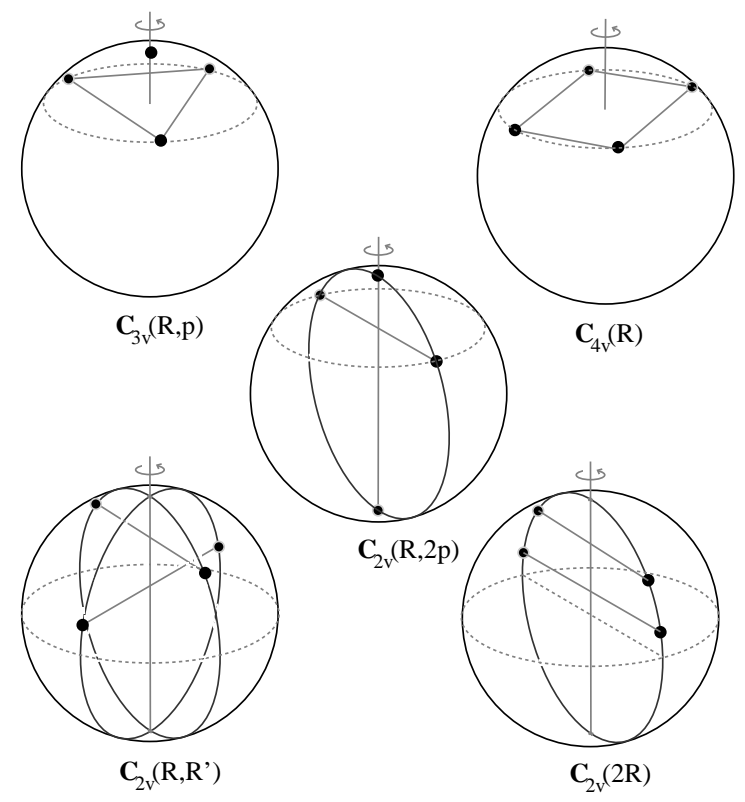

Figure 8. Relative equilibria for 4 identical vortices on the sphere

function theorem one can show that many of the REs shown to exist in [37] persist under small perturbations of the Hamiltonian, and so in particular under small changes of the values of the vorticities. There is one interesting occurrence of a symmetric pitchfork bifurcation: consider the family of square configurations, on the co-latitude $\theta$-type $\mathbf{C}_{4 v}(R)$ in the figure. When the ring is close to the North pole (say), the RE is stable. As $\theta$ increases, one pair of eigenvalues approaches 0 , and at $\theta=\arccos (1 / \sqrt{3})$ there is a pitchfork bifurcation (of type I in the terminology of $\S 4.1$ ). The bifurcating pair of relative equilibria are of type $\mathbf{C}_{2 v}\left(R, R^{\prime}\right)$.

Stability of a ring of vortices It was shown by Dritschel and Polvani [64] that the stability of a single ring of identical vortices depends on the latitude. They show that if $\theta$ is the angle subtended by any of the vortices with the axis of symmetry of the ring (the colatitude), then the configuration of a regular 
ring of $N$ identical vortices is linearly stable as follows

\begin{tabular}{|c|c||c|c|}
\hline$N$ & range of stability & $N$ & range of stability \\
\hline 3 & all $\theta$ & 4 & $\cos ^{2} \theta>1 / 3$ \\
5 & $\cos ^{2} \theta>1 / 2$ & 6 & $\cos ^{2} \theta>4 / 5$ \\
\hline
\end{tabular}

while for $N>6$ the ring is never stable. See also [38], where it is shown that the rings are not only linearly stable but Lyapounov stable.

Bifurcations The changes in stability that occur in the table above involve bifurcations of the relative equilibria, and indeed the supercritical pitchfork bifurcation described in Section 4, since they all involve a loss of $\mathbf{C}_{2}$ symmetry. Consider for example the case of $N=4$ identical vortices. A single ring (square) near the pole is a Lyapounov stable relative equilibrium. As $\theta \rightarrow \cos ^{-1}(1 / \sqrt{3})$, so one of the eigenvalues tends to 0 , and for $\theta>\cos ^{-1}(1 / \sqrt{3})$, there appears a new family of relative equilibria consisting of vortices alternately above and below the vortices in the now-unstable square configuration. These bifurcating REs - denoted $\mathbf{C}_{2 v}\left(R, R^{\prime}\right)$ in [37] - are then Lyapounov stable. Other stability transitions have been observed in $[37,38]$, but the corresponding bifurcations have not been studied.

The other type of bifurcation that occurs in this problem is the geometric bifurcation due to the different geometry of the reduced spaces for $\mu=0$ and $\mu \neq 0$ - see Section 5. Consider a relative equilibrium $p_{e}$ on $\mu=0$, for example the ring of $N$ identical vortices on the equator. This corresponds to a point with symmetry $D_{N h}$ in the phase space (the dihedral group in the equatorial plane together with inversion in that plane). Nearby reduced spaces are then locally of the form $\mathcal{P}_{\mu} \simeq \mathcal{P}_{0} \times \mathcal{O}_{\mu}$, where $\mathcal{O}_{\mu}$ is the coadjoint orbit through $\mu$, which here is a sphere, as described very briefly in Section 5 . The relative equilibria on $\mathcal{P}_{\mu}$ near $p_{e}$ are the critical points of some function $h: \mathcal{O}_{\mu} \rightarrow \mathbf{R}$, and moreover this function is invariant under some action of $\mathbf{D}_{n h} \simeq \mathbf{D}_{N} \times \mathbf{C}_{2}$ on $\mathcal{O}_{\mu}$. An analysis of this action shows that there must be critical points with symmetry of types $\mathbf{C}_{N v}$ and $\mathbf{C}_{2 v}$; see Figure 9 . The corresponding relative equilibria have configurations of types $\mathbf{C}_{N v}(R)$ (a regular ring) and if $N=2 m$ then $\mathbf{C}_{2 v}(m R)$ and $\mathbf{C}_{2 v}((m-1) R, 2 p)$, while if $N=2 m+1$ then $\mathbf{C}_{2 v}(m R, p)$. Here the configuration $\mathbf{C}_{2 v}(m R, \ell p)$ consists of $m$ pairs and $\ell$ poles all lying on a common great circle, while the great circle is rotating rigidly about an axis containing the poles. See Figure 8, and see [37] for details.

\subsection{Point vortices in the plane}

This system has a much older history than the model of vortices on the sphere, going back to Helmoltz and Kirchhoff, and has been studied by many people

J. Montaldi, Relative equilibria and conserved quantities .... 


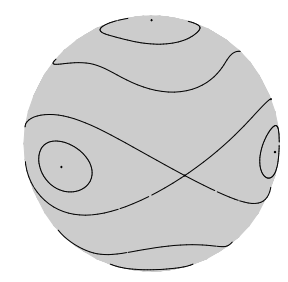

Figure 9. Level sets of a typical function on a sphere with symmetry $\mathbf{D}_{3 h}$, showing half of the 8 critical points

since; for reviews see $[17,3,15]$. Consider an ideal fluid in the plane whose vorticity is concentrated in $N$ point vortices, of strengths $\lambda_{1}, \ldots, \lambda_{N}$. These points move according to the differential system

$$
\dot{\bar{z}}_{j}=\frac{1}{2 \pi i} \sum_{k \neq j} \frac{\lambda_{k}}{z_{j}-z_{k}},
$$

where $z_{j}$ is a complex number representing the position of the $j$-th vortex, after identifying the plane with $\mathbf{C}$, see (1.3). The Hamiltonian for this system is

$$
H(\mathbf{z})=-\frac{1}{4 \pi} \sum_{j<k} \lambda_{j} \lambda_{k} \log \left|z_{j}-z_{k}\right|^{2}
$$

The symmetry here is the group of 2-D Euclidean motions $\mathbf{S E}(2)$ - which is not compact. The corresponding conserved quantity (momentum map) is

$$
\Phi\left(z_{1}, \ldots, z_{N}\right)=\left(i \sum_{j} \lambda_{j} z_{j}, \frac{1}{2} \sum \lambda_{j}\left|z_{j}\right|^{2}\right) .
$$

From the geometric point of view, this is interesting because if the total vorticity $\Lambda \equiv \sum_{j} \lambda_{j}$ is non-zero, the coadjoint action on $\mathfrak{s e}(2)^{*}$ must be modified in order that the momentum map be equivariant (see Section 2). If we identify $\mathbf{S E}(2)$ with $\mathbf{C} \rtimes \mathbf{U}(1)$ and $\mathfrak{u}(1)$ with $\mathbf{R}$, then the modified coadjoint action (2.7) becomes

$$
\operatorname{Coad}_{(u, \theta)}^{\Lambda}(\nu, \psi)=\left(e^{i \theta} \nu, \psi+\Im\left(e^{i \theta} \nu \bar{u}\right)\right)+\Lambda\left(i u, \frac{1}{2}|u|^{2}\right)
$$

where $\Im(z)$ is the imaginary part of $z$. 
If $\Lambda=0$ then the orbits are points on the $\psi$-axis, and cylinders around that axis, while if $\Lambda \neq 0$ the orbits are all paraboloids - see Examples 2.5 and 2.7 respectively and Figures 2 and 3. Indeed, one can show that the modified coadjoint orbits are given by the level sets of $f: \mathbf{C} \times \mathbf{R} \rightarrow \mathbf{R}$ defined by

$$
f(\nu, \psi)=|\nu|^{2}-2 \Lambda \psi,
$$

at least if $\Lambda \neq 0$. If $\Lambda=0$ then the non-zero level sets are the cylindrical orbits, but the zero level set is the whole $\psi$-axis.

2 vortices in the plane As in the case of 2 vortices on the sphere, here they are also always relative equilibria. It is simple to prove from the differential equation that if $\Lambda \equiv \lambda_{1}+\lambda_{2} \neq 0$ then the two point vortices rotate about the fixed point $\left(\lambda_{1} z_{1}+\lambda_{2} z_{2}\right) / \Lambda$. On the other hand, if $\Lambda=0$ then they translate together towards infinity, in the direction orthogonal to the segment joining them. These two types of motion are both relative equilibria.

From a geometrical point of view, the reduced spaces are all just single points, so the corresponding motions are indeed all relative equilibria, and furthermore the relative equilibria are trivially extremal, and so provided $G_{\mu}$ is compact (ie. $\Lambda \neq 0$, so $G_{\mu} \simeq \mathbf{S O}(2)$ ) they are stable modulo $G_{\mu}$.

3 vortices in the plane The classical work on three planar point vortices is a beautiful paper by J.L. Synge [69]. We approach this problem as we did for three point vortices on the sphere. That is, points in the quotient of the phase space $\left(\mathbf{R}^{2}\right)^{3} \simeq \mathbf{C}^{3}$ by $\mathbf{S E}(2)$ correspond to shapes of oriented triangles. Again we ignore the orientation, which only causes problems near collinear configurations. Then a point in the quotient space is determined by the three lengths $\left(r_{1}, r_{2}, r_{3}\right)$, and on that space

$$
\begin{aligned}
4 \pi H\left(r_{1}, r_{2}, r_{3}\right) & =-\lambda_{1} \lambda_{2} \log \left(r_{3}\right)-\lambda_{2} \lambda_{3} \log \left(r_{1}\right)-\lambda_{3} \lambda_{1} \log \left(r_{2}\right) \\
\varphi\left(r_{1}, r_{2}, r_{3}\right) & =-\lambda_{1} \lambda_{2} r_{3}^{2}-\lambda_{2} \lambda_{3} r_{1}^{2}-\lambda_{3} \lambda_{1} r_{2}^{2},
\end{aligned}
$$

the second is just $f \circ \Phi$. It is remarkable that apart from constant term in $\varphi$, these are identical to equations (6.1) for 3 point vortices on the sphere.

The non-collinear relative equilibria are given by the critical points of $H$ restricted to the level-sets of $\varphi$, and the computation has already been done. Thus the relative equilibria are again equilateral triangles, of side $r$ say, with Lagrange multiplier $\eta=1 /\left(4 \pi r^{2}\right)$ again. Note however, that this time the relation between $\eta$ and the "angular velocity" $\xi$ is not so simple:

$$
0=d(H-\eta \varphi)(p)=d(H-\eta f \circ \Phi)(p)=d H(p)-\eta d f(\mu) d \Phi(p),
$$

so that $\xi=\eta d f(\mu)$. Thus if $\Phi(p)=(\nu, \psi)$ then

$$
\xi=2 \eta(\nu,-\Lambda) \text {. }
$$


One consequence of this expression for $\xi$ is that if $\Lambda=0$ then the "angular velocity" is in fact rectilinear motion, with constant velocity $\xi=2 \eta \nu=$ $\left(1 / 2 \pi r^{2}\right) \nu$, where $\nu=i \sum_{j} \lambda_{j} z_{j}$, which in modulus is independent of the representative triangle. Note that if the 3 vortices are not collinear, then $\nu \neq 0$ so that the function $f$ separates the relevant coadjoint orbits even in the case $\Lambda=0$. On the other hand, if $\Lambda \neq 0$, and $\eta \neq 0$, then the relative equilibrium is a periodic orbit.

Furthermore, the Lyapounov stabilities modulo $G$ of these equilateral relative equilibria are the same as for the spherical case.

The symplectic reduction for a particular class of planar 4-vortex problems has recently been considered by Patrick [61]. In particular he treats the case $\Lambda=0$ so that the momentum map is coadjoint-equivariant; it would be interesting to see how the results are affected by changing to $\Lambda \neq 0$.

\subsection{Molecules}

Consider a molecule consisting of $N$ atoms. The Born-Oppenheimer approximation consists of ignoring the movement of the electrons (which is reasonable as they are so light). The system then has $3 N$ degrees of freedom, the 3 directions of motion for each nucleus, or $3 N-3$ after fixing the centre of mass.

The rotational symmetry of the system gives rise to the conservation of angular momentum $\mathbf{J}$. If we put $\mathbf{J}=0$, then there are $3 N-6$ degrees of freedom which describes the shape of the molecule, and correspond to the vibrational motions. In other words, the $\mathbf{J}=0$ reduced space is of dimension $6 N-12$. The simplest motions beyond the equilibria are the periodic orbits, which near the stable equilibrium are given by Lyapounov's theorem and its generalizations. For $\mathbf{J} \neq 0$, the motion has a rotational aspect, and the simplest type of motion is the relative equilibrium. Beyond that are motions that are a combination of rotations and vibrations, so-called rovibrational states. The reduced spaces for $\mathbf{J} \neq 0$ are of dimension $6 N-10$.

Consider now the simplest interesting case of a triatomic molecule. The reduced spaces are of dimension 6 (for $\mathbf{J}=0$ ) or 8 (for $\mathbf{J} \neq 0$ ). There is one complication that we will not discuss here, namely the reduced space for $\mathbf{J}=0$ is singular at points corresponding to collinear equilibria. Consider then an equilibrium of a triatomic molecule which is not collinear. The geometric bifurcation methods discussed in Section 5, and at the end of $\S 6.1$, show that there are relative equilibria which bifurcate from the equilibria, and in fact there at at least 6 such families of RE parametrized by $\|\mathbf{J}\|$ and corresponding to critical points of functions on a sphere. The stabilities of these bifurcating families are discussed in [50]. For a complete investigation into the relative 
equilibria of a specific molecule with 3 identical atoms — namely $\mathrm{H}_{3}^{+}$— see [33].

Another interesting example is the tetra-atomic molecule ammonia $\mathrm{NH}_{3}$. This has an equilibrium where the three hydrogen atoms form an equilateral triangle, and the nitrogen atom is slighly above (or below) the centre of the triangle - so that the molecule is nearly planar. The analysis is similar to the triatomic case, with 6 families of REs that bifurcate from each equilibrium. The stability analyses should also be similar to the triatomic case. However, the presence of two very close stable equilibria (with the nitrogen atom on one side and on the other of the hydrogen-plane) and an unstable planar equilibrium betewen them suggests that there will be further bifurcations. An interesting "semilocal" analysis could be obtained by adding a new parameter $\lambda$ so that the equilibria undergo a pitchfork bifurcation (of type I) at $\lambda=0$, with the genuine system corresponding to say $\lambda=-1$ and an artificial one for $\lambda>0$ with the symmetric planar equilibrium being stable. One needs to investigate how the pitchfork bifurcation within the reduced space $\mathcal{P}_{0}$, obtained by varying $\lambda$, couples with the geometric bifurcation, obtained by varying $\|\mathbf{J}\|$.

\section{References}

Books

1. R. Abraham and J. Marsden. Foundations of Mechanics. BenjaminCummings, 1978.

2. V.I. Arnold. Mathematical Methods of Classical Mechanics. SpringerVerlag, 1978.

3. V.I. Arnold and B.A. Khesin, Topological Methods in Hydrodynamics, Springer-Verlag, New York, 1998.

4. V.I. Arnold, V.V. Kozlov and A.I. Neishtadt. Mathematical Aspects of Classical and Celestial Mechanics, $2^{\text {nd }}$ edition. Springer-Verlag, 1997.

5. P. Chossat and R. Lauterbach, Methods in Equivariant Bifurcation Theory and Dynamical Systems. World Scientific, to appear 2000.

6. R.H. Cushman and L.M. Bates, Global Aspects of Classical Integrable Systems. Birkhuser Verlag. 1997.

7. M. Golubitsky, I. Stewart and D. Schaeffer. Singularities and Groups in Bifurcation Theory, Vol. II. Springer-Verlag, New York. 1988.

8. V. Guillemin and S. Sternberg Symplectic Techniques in Physics. Cambridge University Press. 1984.

9. V. Guillemin, E. Lerman and S. Sternberg Symplectic Fibrations and Multiplicity Diagrams. Cambridge University Press. 1996.

J. Montaldi, Relative equilibria and conserved quantities .... 
10. P. Libermann and C.-M. Marle. Symplectic Geometry and Analytical Mechanics. Reidel, 1987.

11. R. MacKay and J. Meiss. Hamiltonian Dynamical Systems, a reprint collection. Adam Hilger, Bristol. 1988.

12. J. Marsden. Lectures on Mechanics. L.M.S. Lecture Note Series 174, Cambridge University Press, 1992.

13. J. Marsden and T. Ratiu. Introduction to Mechanics and Symmetry. Springer-Verlag, New-York, 1994. [Second ed. 1999]

14. K. Meyer and G. Hall. Hamiltonian Systems and the N-Body Problem. Springer-Verlag, New-York, 1992.

15. P.G. Saffman, Vortex Dynamics. Cambridge University Press, Cambridge, 1992.

16. J.-M. Souriau Structure des Systèmes Dynamiques. Dunod, Paris, 1970. [English translation: Structure of Dynamical Systems: A Symplectic View of Physics, Birkhauser, Boston, 1997.]

Research papers

17. H. Aref, Integrable, chaotic and turbulent vortex motion in twodimensional flows, Ann. Rev. Fluid Mech. 15 (1983), 345-389.

18. J.M. Arms, A. Fischer and J.E. Marsden, Une aproche symplectique pour des théorèmes de décomposition en géométrie ou relativité générale. $C$. R. Acad. Sci. Paris 281 (1975), $517-520$.

19. J.M. Arms, J.E. Marsden and V. Moncrief, Bifurcations of momentum mappings. Comm. Math. Phys. 78 (1981), 455-478.

20. L.M. Bates and E. Lerman, Proper group actions and symplectic stratified spaces. Pacific J. Math. 181 (1997), 201-229.

21. V.A. Bogomolov, Dynamics of vorticity at a sphere, Fluid Dynamics 6 (1977), 863-870.

22. H. Broer, S.-N. Chow, Y. Kim and G. Vegter, A normally elliptic Hamiltonian bifurcation. Z. Angew. Math. Phys. 44 (1993), 389-432.

23. M. Dellnitz, I. Melbourne and J. Marsden, Generic bifurcation of Hamiltonian vector fields with symmetry. Nonlinearity 5 (1992), 979-996.

24. J.J. Duistermaat, Bifurcations of perodic solutions near equilibrium points of Hamiltonian systems. In Bifurcation Theory and Applications, Montecatini, 1983 (ed. L. Salvadori), LNM 1057, Springer, 1984.

25. F. Fassò and D. Lewis, Stability properties of the Riemann ellipsoids. Preprint, 2000.

26. I.M. Gelfand and L.D. Lidskii, On the structure of stability of linear Hamiltonian systems of differential equations with periodic coefficients. 
Usp. Math. Nauk. 10 (1955), 3-40. (English translation: Amer. Math. Soc. Translations (2) 8 (1958), 143-181.)

27. M. Golubitsky and I. Stewart, Generic bifurcations of Hamiltonian systems with symmetry. Physica D 24 (1987), 391-405.

28. V. Guillemin and S. Sternberg, A normal form for the moment map. In Differential Geometric Methods in Mathematical Physics (S. Sternberg ed.) Mathematical Physics Studies, 6. D. Reidel Publishing Company (1984).

29. G. Iooss and M.-C. Pérouème, Perturbed homoclinic solutions in reversible 1:1 resonance vector fields. J. Differential Equations 102 (1993), $62-88$.

30. R. Kidambi and P. Newton, Motion of three point vortices on a sphere, Physica D 116 (1998), 143-175.

31. Y. Kimura, Vortex motion on surfaces with constant curvature. Proc. R. Soc. Lond. A 455 (1999), 245-259.

32. F.C. Kirwan, The topology of reduced phase spaces of the motion of vortices on a sphere, Physica D 30 (1988), 99-123.

33. I. Kozin, R.M. Roberts and J. Tennyson, Symmetry and structure of rotating $\mathrm{H}_{3}^{+}$. Preprint, University of Warwick, 1999.

34. E. Lerman, J. Montaldi and T. Tokieda, Persistence of extremal relative equilibria. In preparation

35. E. Lerman and S.F. Singer, Relative equilibria at singular points of the momentum map. Nonlinearity 11 (1998), 1637-1649

36. E. Lerman and T.F. Tokieda, On relative normal modes. C. R. Acad. Sci. Paris Sr. I 328 (1999), 413-418.

37. C.C. Lim, J. Montaldi and R.M. Roberts, Systems of point vortices on the sphere. Preprint, INLN, 2000.

38. C.C. Lim, J. Montaldi and R.M. Roberts, Stability of relative equilibria for point vortices on the sphere. In preparation.

39. E. Lombardi, Oscillatory integrals and phenomena beyond any algebraic order; with applications to homoclinic orbits in reversible systems. Springer Verlag Lecture Notes in Mathematics. To appear.

40. A.M. Lyapounov, Problème générale de la stabilité du mouvement. Ann. Fac. Sci. Toulouse 9, (1907). (Russian original: 1895.)

41. R. MacKay, Stability of equilibria of Hamitonian systems. Nonlinear Phenomena and Chaos (1986), 254-70. Also reprinted in [11].

42. C.-M. Marle, Modèle d'action hamiltonienne d'un groupe the Lie sur une variété symplectique. Rend. Sem. Mat. Univers. Politecn. Torino 43 (1985), 227-251.

43. J. Marsden and A. Weinstein, Reduction of symplectic manifolds with 
symmetry. Pep. Math. Phys 5 (1974), 121-130.

44. J.-C. van der Meer, The Hamiltonian Hopf bifurcation, Lecture Notes in Math. 1160, Springer, 1985.

45. I. Melbourne, Versal unfoldings of equivariant linear Hamiltonian vector fields. Math. Proc. Camb. Phil. Soc. 114 (1993), 559-573.

46. I. Melbourne and M. Dellnitz, Normal forms for linear Hamiltonian vector fields commuting with the action of a compact Lie group. Math. Proc. Camb. Phil. Soc. 114 (1993), 235-268.

47. K. Meyer, Symmetries and integrals in mechanics. Dynamical Systems (M. Peixoto, ed.), 259-273. Academic Press, New York, 1973.

48. J. Montaldi, Persistence and stability of relative equilibria. Nonlinearity 10 (1997), 449-466.

49. J. Montaldi, Perturbing a symmetric resonance: the magnetic spherical pendulum. In SPT98 - Symmetry and Perturbation Theory II, (A. Degasperis and G. Gaeta eds.), World Scientific 1999.

50. J. Montaldi and R.M. Roberts, Relative equilibria of molecules. J. Nonlin. Sci. 9 (1999), 53-88.

51. J. Montaldi and R.M. Roberts, A note on semisymplectic actions of Lie groups. In preparation.

52. J. Montaldi, R.M. Roberts and I. Stewart, Periodic Solutions near Equilibria of Symmetric Hamiltonian Systems. Proc. Roy. Soc. London 325 (1988), 237-293.

53. J. Montaldi, R.M. Roberts and I. Stewart, Existence of nonlinear normal modes of symmetric Hamiltonian systems. Nonlinearity 3 (1990), 695730 .

54. J. Moser, Lectures on Hamiltonian Systems. Memoirs of the A.M.S. 81 (1981). (Also reprinted in [11]).

55. J. Moser, Periodic orbits near equilibrium and a theorem by Alan Weinstein. Communs. Pure Appl. Math. 29 (1976), 727-747.

56. J.P. Ortega, Symmetry, Reduction ans Stability in Hamilonian Systems. Thesis, University of California, Santa Cruz, 1988.

57. J.P. Ortega and T.S. Ratiu, Stability of Hamiltonian relative equilibria. Nonlinearity 12 (1999), 693-720.

58. R. Palais. The principle of symmetric criticality, Commun. Math. Phys. 69 (1979), 19-30.

59. G.W. Patrick, Relative equilibria in Hamiltonian systems: The dynamic interpretation of nonlinear stability on the reduced phase space. J. Geom. Phys. 9 (1992), 111-119.

60. G.W. Patrick, Relative equilibria of Hamiltonian systems with symmetry: linearization, smoothness and drift. J. Nonlin. Sci. 5 (1995), 373-418.

J. Montaldi, Relative equilibria and conserved quantities .... 
61. G.W. Patrick, Reduction of the planar 4-vortex system at zero momentum. Preprint math-ph/9910012

62. G.W. Patrick and R.M. Roberts, The transversal relative equilibria of Hamiltonian systems with symmetry. Preprint, University of Warwick (1999).

63. S. Pekarsky and J.E. Marsden, Point vortices on a sphere: Stability of relative equilibria, J. Mathematical Physics 39 (1998), 5894-5906.

64. L.M. Polvani and D.G. Dritschel, Wave and vortex dynamics on the surface of a sphere, J. Fluid Mech. 255 (1993), 35-64.

65. R.M. Roberts and M.E.R. Sousa Dias, Bifurcations from relative equilibria of Hamiltonian systems. Nonlinearity 10 (1997), 1719-1738.

66. R.M. Roberts and M.E.R. Sousa Dias, Symmetries of Riemann ellipsoids. To appear in Resenhas do IME - USP, 2000.

67. R. Sjamaar and E. Lerman, Stratified symplectic spaces and reduction. Ann. Math 134 (1991), 375-422.

68. S. Smale, Topology and mechanics I, II. Invent. Math. 10 (1970), 305331, and 11 (1970), 45-64.

69. J.L. Synge, On the motion of three vortices. Can. J. Math. 1 (1949), 257-270.

70. A. Vanderbauwhede and J.C. van der Meer, A general reduction method for periodic solutions near equilibria in Hamiltonian systems. Fields Institute Comm. 4 (1995), 273-294.

71. A. Weinstein, Normal modes for nonlinear Hamiltonian systems. Invent. Math. 20 (1973), 47-57.

72. A. Weinstein, Bifurcations and Hamilton's principle. Math. Z. 159 (1978), 235-248. 